\title{
Simulations of three one-dimensional limits of the strong-strong beam-beam interaction in hadron colliders using weighted macroparticle tracking
}

\author{
M. Vogt* \\ University of New Mexico, Albuquerque, New Mexico 87131 \\ T. Sen ${ }^{\dagger}$ \\ Fermilab, P.O. Box 500, Batavia, Illinois 60510 \\ J. A. Ellison \\ University of New Mexico, Albuquerque, New Mexico 87131
}

(Received 5 June 2001; published 25 February 2002)

\begin{abstract}
We develop the method of weighted macroparticle tracking (WMPT) for simulating the time evolution of the moments of the phase space densities of two beams which are coupled via the collective (strongstrong) beam-beam interaction in the absence of diffusion and damping. As an initial test we apply this method to study the $\pi$ mode and the $\sigma$ mode in three different 1D limits of the beam-beam interaction. The three limits are flat beams and transverse motion in the direction of the small width, round beams, and flat beams and motion in the direction of the large width. We have written a code (BBDeMo1D) based on WMPT, which allows testing of all three limits and is suited for extension to 2 degrees of freedom.
\end{abstract}

DOI: 10.1103/PhysRevSTAB.5.024401

\section{INTRODUCTION}

At high energy hadron colliders the need for high luminosity requires highly focused intense beams at the interaction points (IPs). These high current densities imply a strong interaction of both beams. Therefore the phase space densities of both counterrotating beams become strongly coupled. Under simplifying assumptions, their evolution may be described by an integro partial differential equation (PDE), the Vlasov equation (VE). Various methods can be used to simulate the Vlasov evolution of the phase space densities numerically. The idea of tracking phase space densities to follow beam evolution in nonlinear fields was proposed by Kauffmann et al. [1]. Here we study a method that is based on computing the expectation values of functions on phase space with the time dependent density by "weighted macroparticle tracking" (WMPT). This method, to be explained in more detail in Sec. II B, has the great advantage that it should be easy to implement in every reasonable multiparticle tracking code.

In the strong-strong treatment, the collective force between the two beams depends on the phase space densities. Moreover, the relative strength of the beam-beam interaction depends on the particle species, the beam energies, the beam emittances, the optics at the interaction regions, the number of IPs, and the phase advance between the IPs. Here we study simplified models of the strong-strong case for head-on collisions in 1 spatial degree of freedom and

\footnotetext{
*Email address: vogtm@math.unm.edu

†Email address: tsen@fnal.gov

†Email address: ellison@math.unm.edu
}

PACS numbers: 29.27.Bd, 29.20.Dh, 02.60.Nm, 41.85.Ew

absorb all parameters of the beam-beam interaction into the linear beam-beam tune shift parameter $\xi$. We assume that a self-consistent common closed orbit at the IP exists and that it does not change with time.

Assuming bunches of length large compared to their transverse dimensions but still small compared to the beta functions at the IPs and no crossing angle at the IPs, the beam-beam interaction is intrinsically $2 \mathrm{D}$, i.e., acts on a 4D phase space. Nevertheless, as a first step one might look at various 1D limits which require less computing time than 2D problems. Typically three different limits from 2D to 1D can be considered: (i) flat beam and motion in the "thin" direction, (ii) an axially symmetric beam and motion in a "radial" direction, and (iii) a flat beam and motion in the "thick" direction. The first limit [2] and the third limit $[3,4]$ have been studied analytically and to some extent numerically, and the second limit, while turning out to be basically inconsistent, leads to a beam-beam force that is of the same form as the one used in weak-strong simulations for round beams. When studying the centroid motion of the phase space densities, two modes, the sum ( $\sigma$ mode) and the difference ( $\pi$ mode), have been previously calculated with different characteristic frequencies, depending on the 1D model [2-4] or the 2D aspect ratio $[5,6]$. One task of the initial stage of this study is to identify these two modes and see if a fully nonlinear numerical treatment yields results similar to the analytic approximations made in [2-4]. Moreover, the 2D simulations for equal vertical and horizontal beam sizes performed in [6] can be compared to some extent to the axially symmetric limit of our 1D simulations.

Flat beams seem more suitable for the description of $e^{ \pm}$beams. On the other hand, the assumption of axial symmetry will, in the following, turn out to be too strong 
under the strong-strong premises. Therefore, this first part of our study can be seen only as a starting point for further investigations. For this purpose, one of the authors has developed a numerical code (BBDeMo1D) that allows the simulation of all three above cases, is easily extended to $2 \mathrm{D}$, and offers a wide variety of diagnostic features.

Section II describes the model of the ring (rotate-kick) we will be using throughout this paper and the simulation method (WMPT). Section III defines and describes the three 1D limits. In Sec. IV the accuracy of the method is analyzed in two ways. First, the stability of an equilibrium solution of the linearized beam-beam force under the fully nonlinear evolution is discussed. Second, the effect of the redistribution of the trajectories after many turns on the sampling of the phase space is analyzed. Section V contains results of our simulations with the three limits; in particular, we calculate the dipole mode spectra of the beams and look for the $\pi$ and $\sigma$ modes. We examine the changes in these spectra as the tunes of the two beams are separated and also as the difference in beam-beam parameters is varied. Section VI gives a short summary and outlook. Appendices A and B discuss technical details of the simulation for one of the three limiting 1D cases, and Appendix $\mathrm{C}$ gives a summary of short definitions of the symbols and conventions used in this paper.

\section{THE ROTATE-KICK MODEL}

This preliminary study is restricted to head-on interactions between two short, counterrotating bunches at a given IP and a linear lattice elsewhere.

The VE that governs the beam-beam interaction is formally symmetric in the two densities. Therefore we will use the notation that if $x$ represents some quantity of "one beam", then $x^{*}$ represents the same quantity of "the other beam."

Let $\psi_{\theta}(\vec{z}) \equiv \psi_{\theta}(q, p)$ be the phase space density at azimuth $\theta \in[0, \infty)$ and at the phase space point $\vec{z} \equiv(q, p)$ and $\rho_{\theta}(q):=\int_{\mathbb{R}} \psi_{\theta}(q, p) d p$ the density in configuration space, both normalized so that $\int_{\mathbb{R}^{2}} \psi_{\theta} d^{2} z=\int_{\mathbb{R}} \rho_{\theta} d q=$ 1 for all $\theta$. Let $\theta_{c}$ denote the azimuth at the collision point. Then the one turn map for turn $m$ to $m+1$ at the azimuth $\theta_{c}^{+}+m 2 \pi$ directly after the IP is

$$
\vec{T}_{m}=\vec{K}\left[\rho_{\theta_{c}^{-}+m 2 \pi}^{*}\right] \circ \vec{R}
$$

where $\vec{K}\left[\rho_{\theta_{c}^{-}+m 2 \pi}^{*}\right]$ is the map for the nonlinear beambeam kick due to the collective force which depends on the spatial density $\rho_{\theta_{c}^{-}}^{*}$ of the other beam directly before the IP and $\vec{R}(\vec{z})=\underline{R} \vec{z}, \underline{R} \in \mathbf{S P}(2)$ is the linear map from $\theta_{c}^{+}$to $\theta_{c}^{-}$of the rest of the lattice. We will often call this the rotate-kick model. In the following we will suppress the azimuth advance $m 2 \pi$ from $\theta_{0}=0$ to $m 2 \pi$ in the subscripts of $\rho$ and $\psi$ and abbreviate $\theta_{c}^{-}+m 2 \pi$ with $\theta_{c}^{-}$ where the possible variation from turn to turn is implicitly understood. The beam-beam kick is explicitly

$$
\begin{aligned}
\vec{K}\left[\rho_{\theta_{c}^{-}}^{*}\right](q, p) & =\left(\begin{array}{c}
q \\
p+K\left[\rho_{\theta_{c}^{-}}^{*}\right](q)
\end{array}\right), \\
K\left[\rho_{\theta_{c}^{-}}^{*}\right](q) & :=\zeta \int_{\mathbb{R}} G\left(q, q^{\prime}\right) \rho_{\theta_{c}^{-}}^{*}\left(q^{\prime}\right) d q^{\prime},
\end{aligned}
$$

with some model dependent kernel $G\left(q, q^{\prime}\right)$ and some strength parameter $\zeta$. It seems reasonable to assume that $\alpha_{0}=0$ at the IP for the unperturbed linear lattice, and hence

$$
\underline{R}=\left(\begin{array}{cc}
\cos 2 \pi Q_{0} & \beta_{0} \sin 2 \pi Q_{0} \\
-\beta_{0}^{-1} \sin 2 \pi Q_{0} & \cos 2 \pi Q_{0}
\end{array}\right) .
$$

We can now precisely define our model for the evolution of the phase space density $\psi$. Because the one turn map $\vec{T}_{m}$ is symplectic, conservation of particles gives

$$
\psi_{\theta_{c}^{+}+(m+1) 2 \pi}(\vec{z})=\psi_{\theta_{c}^{+}+m 2 \pi}\left[\vec{T}_{m}^{-1}(\vec{z})\right] .
$$

When we discuss the accuracy of WMPT we mean relative to the density as defined by Eq. (4). This can be viewed as the solution of the associated VE to be discussed in the next section. However, the VE is not well defined with a $\delta$-function kick as we mention in the next section.

Before proceeding we discuss the beam-beam tune shift parameter constructed by linearizing the beam-beam kick. The spatial coordinates of the two beam centroids are $\langle q\rangle=\int_{\mathbb{R}} q \rho d q$ and $\langle q\rangle^{*}=\int_{\mathbb{R}} q \rho^{*} d q$. One may linearize the beam-beam kick around $q=0$ for head-on collisions $\left(\langle q\rangle=\langle q\rangle^{*}=0\right)$ with symmetric densities $\left[\vec{K}\left[\rho_{\theta_{c}^{-}}^{*}\right](0, p)=0\right]$ yielding $\vec{K}\left[\rho_{\theta_{c}^{-}}^{*}\right](\vec{z})=\underline{K} \vec{z}+O\left(\vec{z}^{2}\right)$, where

$$
\underline{K}:=\left(\begin{array}{cc}
1 & 0 \\
\kappa\left[\rho_{\theta_{c}^{-}}^{*}\right] & 1
\end{array}\right), \quad \kappa\left[\rho_{\theta_{c}^{-}}^{*}\right]:=\left.\frac{d}{d q} K\left[\rho_{\theta_{c}^{-}}^{*}\right]\right|_{q=0} .
$$

The Jacobian of the one turn map in Eq. (1) is then $\underline{T}=$ $\underline{K} \underline{R}$, and a stable solution of the linearized motion exists if and only if $\left|\cos 2 \pi Q_{0}+\frac{\kappa \beta_{0}}{2} \sin 2 \pi Q_{0}\right|<1$. Then, using the Courant-Snyder functions of the linearly perturbed lattice $\alpha, \beta, \gamma$ and the linearly perturbed tune $Q$, the Jacobian of the one turn map can be brought to the standard form

$$
\underline{T}=\left(\begin{array}{cc}
\cos 2 \pi Q+\alpha \sin 2 \pi Q & \beta \sin 2 \pi Q \\
-\gamma \sin 2 \pi Q & \cos 2 \pi Q-\alpha \sin 2 \pi Q
\end{array}\right),
$$

where

$$
\begin{aligned}
Q & =\frac{1}{2 \pi} \arccos \left(\cos 2 \pi Q_{0}+\frac{\kappa \beta_{0}}{2} \sin 2 \pi Q_{0}\right) \\
& =Q_{0}-\frac{\kappa \beta_{0}}{4 \pi}+O\left(\kappa^{2} \beta_{0}^{2}\right),
\end{aligned}
$$




$$
\begin{gathered}
\beta=\beta_{0} \frac{\sin 2 \pi Q_{0}}{\sin 2 \pi Q}, \quad \alpha=-\frac{\kappa}{2} \beta, \\
\gamma=\frac{1}{\beta}+\frac{\kappa^{2} \beta}{4} .
\end{gathered}
$$

This analysis contains the parameter $\kappa$ which depends on $\rho_{\theta_{c}^{-}+m 2 \pi}^{*}$ which in principle must be assumed to be changing from turn $m$ to turn $m+1$ because of the nonlinear collective beam-beam interaction. Nevertheless, it gives an intuitive interpretation of the initial strength of a beam-beam interaction in the sense that the beam-beam tune shift parameter $\xi$ is defined by

$$
\begin{aligned}
\left.Q\right|_{m=0}-Q_{0} & =\xi+O\left(\kappa^{2} \beta^{2}\right), \\
\xi & :=-\frac{\kappa\left[\rho_{\theta_{c}^{-}}^{*}\right] \beta_{0}}{4 \pi} .
\end{aligned}
$$

Note that a positive (defocusing) $\kappa$, as it is in the case of $p p$ interactions, results in a negative tune shift parameter.

\section{A. The Vlasov equation}

In the absence of damping and diffusion the phase space densities $\psi_{\theta}$ and $\psi_{\theta}^{*}$ evolve according to the coupled Vlasov equations

$$
\begin{array}{r}
\partial_{\theta} \psi+\partial_{q} \psi \partial_{p} H-\partial_{p} \psi \partial_{q} H=0, \\
\partial_{\theta} \psi^{*}+\partial_{q^{*}} \psi^{*} \partial_{p^{*}} H-\partial_{p^{*}} \psi^{*} \partial_{q^{*}} H=0,
\end{array}
$$

where

$$
\begin{aligned}
H\left[\psi, \psi^{*}\right]\left(\vec{z}, \vec{z}^{*}, \theta\right)= & H_{0}(\vec{z}, \theta)+H_{0}^{*}\left(\vec{z}^{*}, \theta\right) \\
& +\delta_{2 \pi}\left(\theta-\theta_{c}\right) \\
& \times\left(H_{1}\left[\psi_{\theta_{c}^{-}}^{*}\right](\vec{z})+H_{1}^{*}\left[\psi_{\theta_{c}^{-}}\right]\left(\vec{z}^{*}\right)\right)
\end{aligned}
$$

is the Hamiltonian including the collective force and $\delta_{2 \pi}$ is the $2 \pi$-periodic $\delta$ function assuming one IP. Note that $H_{1}$ depends on the densities directly before the collision. This Hamiltonian is equivalent to the rotate-kick model (4) we are going to use. If $H_{1}$ contained $\psi_{\theta}\left(\right.$ or $\left.\psi_{\theta}^{*}\right)$, the resulting VE would not be well defined since the $\delta$ function would be multiplied with $\partial_{q} H_{1}$, which is discontinuous at $\theta \bmod 2 \pi=\theta_{c}$. However, in a real accelerator the beam-beam interaction takes place over a small finite range in azimuth and the $\delta$ function is replaced by a regular distribution in $\theta$. A finite interaction length would then lead to a thick-lens representation of the beam-beam interaction. Moreover, note that the asterisks on $H_{0}$ and $H_{1}$ reflect the fact that in our model "the other beam" may have a different unperturbed tune $Q_{0}^{*}$ and beam-beam tune shift parameter $\xi^{*}$.

In principle, one needs to solve the VE in order to obtain complete knowledge of the coupled multiparticle system. Numerous analytical approximations and numerical simulation methods exist and have been applied to the strong-strong beam-beam interaction. We mention only some of the numerical approaches: (i) standard PDE solvers (divided difference schemes), (ii) particle-in-cell (PIC) codes [7,8], (iii) the Perron-Frobenius (PF) operator method $[9,10]$, and (iv) weighted macroparticle tracking (yields moments of the distribution). The first two methods are well known and will not be discussed here.

The PF operator method employs the conservation of the phase space density along trajectories according to Eq. (4). Given a map $\vec{T}$, the action of the PF operator $\mathfrak{I}$ on a density $\psi$ is simply

$$
\mathfrak{I} \psi(\vec{z})=\psi\left(\vec{T}^{-1}(\vec{z})\right),
$$

and thus Eq. (4) yields

$$
\psi_{\theta_{c}^{+}+(m+1) 2 \pi}=\mathfrak{T} \psi_{\theta_{c}^{+}+m 2 \pi} .
$$

The action of $\mathfrak{I}$ is completely defined by the map $\vec{T}$. Now the densities and $\mathfrak{I}$ are discretized on a square $n \times n$ grid in phase space, the density being defined at off-grid points by local polynomial interpolation. The kick is calculated at grid points from values of the density at grid points. Note that the kick $\vec{K}$ depends only on $q$ and that the kernel $K$ acts on the spatial density $\rho(q)$ so that the computation of the kick for all $N:=n^{2}$ grid points is obtained by the multiplication of an $n$ vector with an $n \times n$ matrix and thus is an $O(N)$ operation. Then $\psi_{\theta_{c}^{+}+m 2 \pi}\left(\vec{T}_{m}^{-1}(\vec{z})\right)$ is computed for grid points $\vec{z}$ by interpolation to give an update of $\psi$ on grid points. Note that the interpolation implies an intrinsic smoothing of the representation of the density on the grid at every step. The PF method has been shown to be stable for the Vlasov-Fokker-Planck equation (using operator splitting to handle the Fokker-Planck part) in beam physics $[9,10]$. Moreover, its main premise, the conservation of the density along trajectories, is also a key concept for the method of WMPT.

\section{B. Weighted macroparticle tracking}

Let $f(\vec{z})$ be a (integrable) function on phase space. Then its average at azimuth $\theta$ is defined as

$$
\begin{aligned}
\langle f\rangle_{\theta} & :=\int_{\mathbb{R}^{2}} f(\vec{z}) \psi_{\theta}(\vec{z}) d^{2} z \\
& =\int_{\mathbb{R}^{2}} f(\vec{z}) \psi_{0}\left(\vec{M}_{\theta}^{-1}(\vec{z})\right) d^{2} z \\
& =\int_{\mathbb{R}^{2}} f\left(\vec{M}_{\theta}(\vec{z})\right) \psi_{0}(\vec{z}) d^{2} z,
\end{aligned}
$$

where $\vec{M}_{\theta}$ is the map from 0 to $\theta$, and where in the third equality we used the fact that the determinant of the Jacobian of a symplectic map is 1 . In this study $f$ is chosen to be either the (sufficiently regular) kernel of the collective force $f_{q}\left(\vec{z}^{\prime}\right):=G\left(q, \mathfrak{X} \vec{z}^{\prime}\right)$, where the projector $\mathfrak{X}$ is defined via $\mathfrak{X} \vec{z}:=q$, or a $(v+w)$ th order monomial $f_{v, w}(\vec{z}):=q^{v} p^{w}$ of the beam distribution. In the first case the average $\left\langle f_{q}\right\rangle_{\theta_{c}^{-}}$is the beam-beam kick exerted by the "unstarred" beam on the "starred" beam, and, in the second 
case, $\left\langle f_{v, w}\right\rangle_{\theta}$ is a $(v+w)$ th order moment of the phase space distribution of the unstarred beam. The averages $\left\langle f_{q}\right\rangle_{\theta_{c}^{-}}^{*}$ and $\left\langle f_{v, w}\right\rangle_{\theta}^{*}$ are analogously defined. Equation (15) means that, in order to compute the expectation value of $f$, it suffices to compute $\left\langle f \circ \vec{M}_{\theta}\right\rangle_{0}$ or, algorithmically speaking, for some representation of $\psi_{0}$ on a (not necessarily square) initial mesh $\left\{\vec{z}_{i j}\right\}_{\substack{1 \leq i \leq n_{q} \\ 1 \leq j \leq n_{p}}}$, an approximation of $\langle f\rangle_{\theta}$ is given,

$$
\langle f\rangle_{\theta} \approx \sum_{i, j=1}^{n_{q}, n_{p}} w_{i j} \psi_{0}\left(\vec{z}_{i j}\right) f\left(\vec{M}_{\theta}\left(\vec{z}_{i j}\right)\right)
$$

where the $w_{i j}$ are the weights of the quadrature formula.

In this study an initially square mesh with $n_{q}=$ $n_{p}=: n, \quad q_{i-1}-q_{i}=\Delta_{q}, \quad p_{j-1}-p_{j}=\Delta_{p}$ for all $i, j$ and the Gaussian midpoint rule have been used so that the quadrature weights are particularly simple: $w_{i j}=: w=\Delta_{q} \Delta_{p}$. Note that once the total weights

$$
\mathfrak{W}_{i j}:=w_{i j} \psi_{0}\left(\vec{z}_{i j}\right)
$$

are assigned to every trajectory

$$
\vec{\eta}_{i j}(\theta):=\vec{M}_{\theta}\left(\vec{z}_{i j}\right)
$$

starting at $\vec{z}_{i j}$, the double index $i j$ can be replaced by one linear index $k$ since the result of a finite sum does not depend on the ordering of the terms. This WMPT procedure requires only forward tracking of macroparticles, and as a by-product to the distribution moments it produces $N:=n^{2}$ particle trajectories and the associated Poincaré sections, etc. Moreover, the conservation of probability is guaranteed by construction [set $f \equiv 1$ in Eq. (16)].

It has been shown for an example of the VE taken from plasma physics [11-13] that, for fixed $\theta$, the trajectories obtained by WMPT converge at least linearly in $\Delta_{q}+\Delta_{p}+\Delta_{\theta}$ to the exact trajectories of the Hamiltonian system Eq. (12). However, the upper bounds on the error given in [11-13] depend exponentially on $\theta$ and are thus not of great use in the case of multiturn tracking.

Finally, it should be mentioned that with WMPT one can obtain an approximation of the phase space density. Let $\left\{\vec{z}_{\mu \nu}:=\left(q_{\mu}, p_{\nu}\right)\right\}_{\substack{1 \leq \mu \leq \tilde{q}_{q} \ll n \\ 1 \leq \nu \leq \tilde{n}_{p} \ll n}}$ be a uniform rectangular mesh and

$$
\chi_{\mu \nu}(\vec{z}):= \begin{cases}1, & q_{\mu}-\frac{\Delta_{q}}{2}<q \leq q_{\mu}+\frac{\Delta_{q}}{2} \quad \text { and } \quad p_{\nu}-\frac{\Delta_{p}}{2}<p \leq p_{\nu}+\frac{\Delta_{p}}{2}, \\ 0, & \text { otherwise }\end{cases}
$$

be the indicator function of the rectangular "bins" $\mathcal{R}_{\mu \nu}:=$ $\left(q_{\mu}-\frac{\Delta_{q}}{2}, q_{\mu}+\frac{\Delta_{q}}{2}\right] \times\left(p_{\nu}-\frac{\Delta_{p}}{2}, p_{\nu}+\frac{\Delta_{p}}{2}\right]$. These indicator functions define a partitioning of unity, in the sense that $\chi_{\mu \nu} \geq 0$ and $\sum_{\mu, \nu} \chi_{\mu \nu}(\vec{z})=1$ for all $\vec{z}$. Then

$$
\begin{aligned}
\left\langle\chi_{\mu \nu}(\vec{z})\right\rangle_{\theta} & =\int_{\mathcal{R}_{\mu \nu}} \psi_{\theta}(\vec{z}) d^{2} z=\int_{\mathbb{R}^{2}} \chi_{\mu \nu}(\vec{z}) \psi_{\theta}(\vec{z}) d^{2} z \\
& \approx \sum_{i, j} \mathfrak{W}_{i j} \chi_{\mu \nu}\left(\vec{\eta}_{i j}(\theta)\right)
\end{aligned}
$$

is an approximation of $\psi_{\theta}$ on the mesh defined by $\left\{\vec{z}_{\mu \nu}\right\}$. The effective smoothness of this approximation depends on the average number of macroparticles in each bin. Therefore the mesh defined by $\vec{z}_{\mu \nu}$ has to be coarser than the initial mesh defined by $\vec{z}_{i j}$. In Sec. IV we will make use of this mesh projection to qualitatively discuss the stability of WMPT. Note that instead of the indicator function $\chi_{\mu \nu}$ we could have used any other partitioning of unity that reflects the mesh structure.

\section{Application of WMPT to the rotate-kick model}

Let $\vec{\eta}_{i j}\left(\theta_{c}^{+}+2 \pi(m-1)\right)$ and $\vec{\eta}_{i j}^{*}\left(\theta_{c}^{+}+2 \pi(m-1)\right)$ be given. Then $\vec{\eta}_{i j}\left(\theta_{c}^{-}+2 \pi m\right)=\underline{R} \vec{\eta}_{i j}\left(\theta_{c}^{+}+2 \pi(m-\right.$ $1))$ and $\vec{\eta}_{i j}^{*}\left(\theta_{c}^{-}+2 \pi m\right)=\underline{R}^{*} \vec{\eta}_{i j}^{*}\left(\theta_{c}^{+}+2 \pi(m-1)\right)$ give the phase space position of the $(i j)$ th particle of each beam just before the kick. The kick on a particle of the unstarred beam at position $q_{i j}:=\mathfrak{X} \vec{\eta}_{i j}\left(\theta_{c}^{-}+2 \pi m\right)$ is given by

$$
\begin{aligned}
K\left[\rho_{\theta_{c}^{-}+2 \pi m}^{*}\right]\left(q_{i j}\right) & =\zeta \int_{\mathbb{R}^{2}} G\left(q_{i j}, \mathfrak{X} \vec{z}^{\prime}\right) \psi_{\theta_{c}^{-}+2 \pi m}^{*}\left(\vec{z}^{\prime}\right) d^{2} z^{\prime}=\zeta \int_{\mathbb{R}^{2}} G\left(q_{i j}, \mathfrak{X} \vec{z}^{\prime}\right) \psi_{0}^{*}\left(\vec{M}_{\theta_{c}^{-}+2 \pi m}^{*-1}\left(\vec{z}^{\prime}\right)\right) d^{2} z^{\prime} \\
& =\zeta \int_{\mathbb{R}^{2}} G\left(q_{i j}, \mathfrak{X} \vec{M}_{\theta_{c}^{-}+2 \pi m}^{*}\left(\vec{z}^{\prime}\right)\right) \psi_{0}^{*}\left(\vec{z}^{\prime}\right) d^{2} z^{\prime} .
\end{aligned}
$$

We then approximate the kick by

$$
K\left[\rho_{\theta_{c}^{-}+2 \pi m}^{*}\right]\left(q_{i j}\right) \approx K\left\{\vec{\eta}^{*}\right\}_{\theta_{c}^{-}+2 \pi m}\left(q_{i j}\right):=\zeta \sum_{k, l=1}^{n} G\left(q_{i j}, \mathfrak{X} \vec{\eta}_{k l}^{*}\left(\theta_{c}^{-}+2 \pi m\right)\right) \mathfrak{W}_{k l}^{*} .
$$

The phase space positions after the kick are thus

$$
\vec{\eta}_{i j}\left(\theta_{c}^{+}+2 \pi m\right)=\vec{K}\left\{\vec{\eta}^{*}\right\}_{\theta_{c}^{-}+2 \pi m}\left(\vec{\eta}_{i j}\left(\theta_{c}^{-}+2 \pi m\right)\right), \quad \vec{\eta}_{i j}^{*}\left(\theta_{c}^{+}+2 \pi m\right)=\vec{K}\{\vec{\eta}\}_{\theta_{c}^{-}+2 \pi m}\left(\vec{\eta}_{i j}^{*}\left(\theta_{c}^{-}+2 \pi m\right)\right),
$$

where $\vec{K}\left\{\vec{\eta}^{*}\right\}_{\theta}$ is the kick map (2) with $K\left[\rho_{\theta_{c}^{-}+2 \pi m}^{*}\right]$ replaced by $K\left\{\vec{\eta}^{*}\right\}_{\theta_{c}^{-}+2 \pi m}$ according to Eq. (22). 
Note that the kicks must be calculated at $N:=n^{2}$ positions $q_{i j}$ and that the calculation of $K\left\{\vec{\eta}^{*}\right\}(q)$ for fixed $q$ takes in principle $N$ evaluations of the kernel $G\left(q_{i j}, \mathfrak{X} \vec{\eta}_{k l}^{*}\right)$ and $N$ multiplications with the total weights $\mathfrak{S}_{k l}$. Therefore the kicks require, in principle, $O\left(N^{2}\right)$ flops where $N$ is the number of macroparticles tracked.

Let $\Delta_{q}=\Delta_{p}=: \Delta$. Then the quadrature error is bounded by $C \Delta^{s}\left\|\vec{M}_{\theta}\right\|$, where $s$ depends on the smoothness of the integrand and the order of the quadrature formula, $\left\|\vec{M}_{\theta}\right\|$ is some derivative norm of $\vec{M}_{\theta}$, and $C$ is a constant independent of $\Delta$ and $\vec{M}_{\theta}$. It is to be expected that $\left\|\vec{M}_{\theta}\right\|$ increases with $\theta$ and so it is natural to ask what the optimal $\Delta$ is for a given interval $\theta \in[0, \Theta]$. Our trajectory calculations indicate that, in a typical setup as in Sec. V, particles which are started inside $1 \sigma_{0}$ fill that region densely. Thus two particles which start $2 \sigma_{0}$ apart can become close, and this can give a jaggedness to an initially smooth density [since trajectories carry their initial density with them; see Eqs. (4), (17), and (18)].

However, the situation is not as bad as it might seem since we are calculating expectation values which potentially can average out any fast oscillating part present in $\vec{M}_{\theta}$. We are investigating optimal $\Delta$ for fixed $\Theta$ and will report that elsewhere.

Finally, we compute phase space averages via

$$
\begin{aligned}
& \langle f\rangle_{\theta} \approx \sum_{i, j} \mathfrak{W}_{i j} f\left(\vec{\eta}_{i j}(\theta)\right), \\
& \langle f\rangle_{\theta}^{*} \approx \sum_{i, j} \mathfrak{W}_{i j}^{*} f\left(\vec{\eta}_{i j}^{*}(\theta)\right) .
\end{aligned}
$$

This is an $O(N)$ calculation and thus is not an important factor in the flop count, as long as the flop count of the kick computation is of higher order in $N$. The quadrature error in the calculation of the averages can be discussed as above; however, the pessimistic view is ameliorated by the inherent smoothing due to integration.

If the integrand can be guaranteed to be sufficiently moderately varying, WMPT, in contrast to methods that require an explicit mesh at each time step, has the advantage that the mesh layout depends only on the initial conditions. As an example, consider a strictly linear one turn map, in particular, no beam-beam interaction and an initial beam distribution with a coherent betatron amplitude with respect to (w.r.t.) the origin (closed orbit) of, say, $x \sigma_{0}$ in normalized coordinates. Then in an explicit mesh method one requires a mesh that is large enough to contain not only the initial distribution up to some reasonable cutoff (say, $\left.c \sigma_{0}\right)$ but to also contain a circle of $(x+c) \sigma_{0}$. Alternatively, if one wants to keep a smaller mesh, one has to recompute the mesh every time step or every few time steps. For the linear case, WMPT has been tested with large initial coherent betatron amplitudes and an initial mesh centered around the beam centroid with width $c \sigma_{0}$. In this case, WMPT carries its mesh along with the trajectories, and the moments computed with WMPT were close to those computed analytically using Eq. (4) to a very high precision.

\section{The Gaussian source approximation}

In the previous section we saw that WMPT might become time consuming due to the loss of the mesh structure and inaccurate due to the buildup of rapid variations in $\psi_{0}(\vec{z}) f\left(\vec{M}_{\theta}(\vec{z})\right)$. These rapid variations would be averaged away in an exact integration but might degrade the accuracy of the result of the numerical quadrature. In particular, errors in the computation of the collective beam-beam kick can become dangerous since they can corrupt the complete dynamics of the coupled two beam system. There is a method [14] that smooths the distribution as far as the collective force is concerned and at the same time makes its computation $O(N)$. The method simply consists of computing some set of moments $\left\{\left\langle q^{k}\right\rangle_{\theta}^{*}\right\}$ of the actual distribution and inserting them as parameters in an analytic formula for the beam-beam kick assuming some test density $\tilde{\rho}$,

$$
\tilde{K}\left(q ;\left\{\left\langle q^{k}\right\rangle_{\theta_{c}^{-}}^{*}\right\}\right):=\int_{\mathbb{R}} G\left(q, q^{\prime}\right) \tilde{\rho}\left(q^{\prime} ;\left\{\left\langle q^{k}\right\rangle_{\theta_{c}^{-}}^{*}\right\}\right) d q^{\prime} .
$$

The form of the starred test density in the computation of the collective form stays fixed-only the change of the parameters reflects the evolution of the density of the other beam. This method is not completely consistent with Vlasov-Poisson evolution since, in general, the form of $\rho_{\theta_{c}^{-}}^{*}(q)$ is different from $\tilde{\rho}\left(q ;\left\{\left\langle q^{k}\right\rangle_{\theta_{c}^{-}}^{*} ;\right\}\right)$. Nevertheless, this method has the benefit of intrinsic smoothing (with suitably chosen $\tilde{\rho}$ ) and allows the simulation of the evolution with a much larger number of macroparticles at a still reasonable computation time. Even then it is still not clear which shape $\tilde{\rho}$ should have, and, in principle, the results to be presented in Sec. IV suggest that it is not a Gaussian shape. There we will see that under the influence of the beam-beam force, the initially Gaussian phase space distributions develop a skewness (third-order centered moment) which is oscillating around zero but with finite amplitude. The core of the density oscillates while the tails are almost stationary. Obviously, a Gaussian-shaped test density cannot represent this skewness properly. Recently, Yokoya [15] showed that a perturbative 2 degrees of freedom model approximating the source term in Poisson's equation by a Gaussian underestimates the phase space averaged beam-beam kick. This leads to a too small frequency shift for the antisymmetric dipole mode ( $\pi$ mode).

However, as the starting point for this study, whenever we approximated the collective kick by Eq. (25), we chose $\tilde{\rho}$ to be a Gaussian,

$$
\tilde{\rho}\left(q ; \mu^{*}, \sigma^{*}\right)=\frac{1}{\sqrt{2 \pi} \sigma^{*}} e^{-\left[\left(q-\mu^{*}\right)^{2} / 2 \sigma^{* 2}\right]} .
$$

Note that $\mu^{*}$ and $\sigma^{*}$ are the actual centroid position w.r.t. the common closed orbit of both beams and width 
of the starred beam, respectively. They have to be updated at each bunch crossing. In the following we will call Eq. (25) with $\tilde{\rho}$ defined by Eq. (26) the Gaussian source approximation (GSA). This approximation is also called the "quasi-strong-strong" or "soft Gaussian" approximation. We want to stress the point that in the GSA the evolution of the densities of the two beams is not faked by some phenomenological evolution law for the parameters $\mu$ and $\nu$ of a Gaussian, but they evolve according to Eq. (4) with a modified collective kick. Only the collective beam-beam kick is approximated. This method (together with tracking an initially Gaussian ensemble of macroparticles of identical weight) has been used in 2D to compute the frequencies of the $\pi$ and $\sigma$ modes with various aspect ratios $\sigma_{x} / \sigma_{y}[6]$.

\section{1D MODELS OF THE BEAM-BEAM KICK}

As pointed out in the Introduction, one may take at least three different limits when breaking down the 2D beam-beam interaction into $1 \mathrm{D}$ models.

(i) The Chao-Ruth (CR) limit [2]. - The beams at the IP are assumed to be flat, e.g., $\sigma_{x} \gg \sigma_{y}$, and the motion is studied in the phase plane associated with $y$,

$$
K_{\mathrm{cr}}\left[\rho_{\theta_{c}^{-}}^{*}\right](y)=\zeta \int_{\mathbb{R}} \operatorname{sgn}\left(y-y^{\prime}\right) \rho_{\theta_{c}^{-}}^{*}\left(y^{\prime}\right) d y^{\prime} .
$$

(ii) The axially symmetric (AS) limit. - The beams are assumed to be round, i.e., $\sigma_{x}=\sigma_{y}$, and the motion w.r.t. an arbitrary transverse direction $(r)$ is studied,

$$
K_{\mathrm{as}}\left[\rho_{\theta_{c}^{-}}^{*}\right](r)=\frac{\zeta}{r} \int_{0}^{r} \rho_{\theta_{c}^{-}}^{*}\left(r^{\prime}\right) r^{\prime} d r^{\prime}
$$

(iii) The Yokoya-Koiso-Zenkevich (YO) limit [3,4].Again, the beams are assumed to be flat, e.g., $\sigma_{x} \gg \sigma_{y}$, but the motion in the phase plane associated with $x$ is studied,

$$
K_{\mathrm{yo}}\left[\rho_{\theta_{c}^{-}}^{*}\right](x)=\zeta f \frac{\rho_{\theta_{c}^{-}}^{*}\left(x^{\prime}\right)}{\left(x-x^{\prime}\right)} d x^{\prime}
$$

where $f$ denotes the Cauchy principal value.

Figure 1 shows the three limits of beam-beam kick $K[\rho](q)$ for a standard Gaussian density $\rho(q ; 0,1)$ and for $\zeta$ chosen so that the slope at the origin is unity (i.e., $\kappa[\rho]=1)$.

We note that the Chao-Ruth limit, as well as the YokoyaKoiso-Zenkevich limit, seems to be more suited for $e^{ \pm}$ colliders.

On the other hand, as it will turn out later, the assumption of axial symmetry is too strong and therefore possibly not a realistic model of two hadron beams coupled by the collective beam-beam force.

In the following three subsections we will discuss the three limits in more detail and, in particular, derive the explicit dependence of the tune shift parameter $\xi$ on the strength parameter $\zeta$ [see Eq. (2)] under the assumption

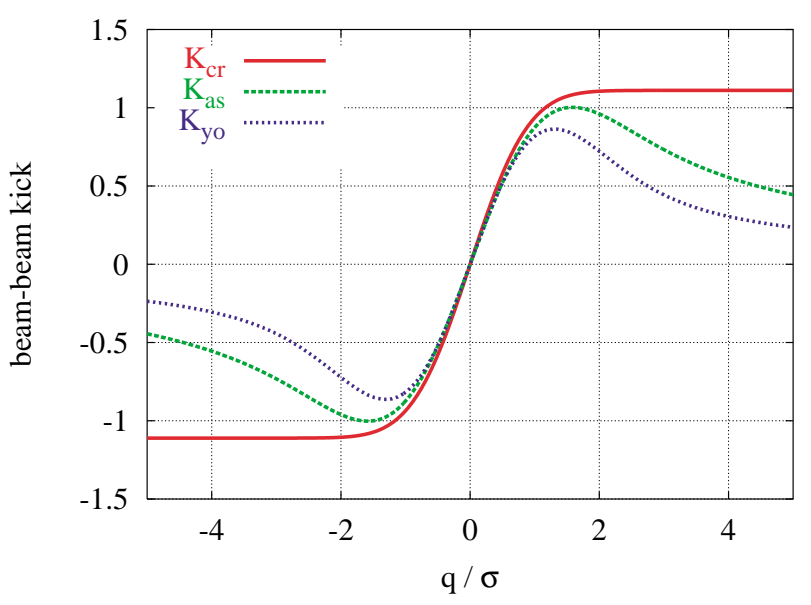

FIG. 1. (Color) The beam-beam kicks $K_{\mathrm{cr}}[\rho](q), K_{\mathrm{as}}[\rho](q)$, and $K_{\mathrm{yo}}[\rho](q)$ for a standard Gaussian $\rho(q ; 0,1)$. In all three cases $\kappa[\rho]$ is chosen to be 1 .

of an initially Gaussian beam. Then $\xi(\zeta)$ can be inverted to obtain the strength for the beam-beam tune shift parameter in the "physical parameters" of a practical collision scheme,

$$
\xi_{x, y}=\frac{r_{p} N^{*} \beta_{x, y}}{2 \pi \gamma_{L} \sigma_{x, y}\left(\sigma_{x}+\sigma_{y}\right)},
$$

where $r_{p}$ is classical particle radius, $\gamma_{L}$ is the Lorentz factor, and $N^{*}$ is the number of particles in the other bunch. Moreover, we will derive the form of the beam-beam kick in the GSA $\tilde{K}\left(q ; \mu_{\theta_{c}^{-}}^{*}, \sigma_{\theta_{c}^{-}}^{*}\right)$.

\section{A. The Chao-Ruth force}

In the Chao-Ruth case, the kick is given by Eq. (27). The Chao-Ruth model can be considered as a 1D Poisson problem $K_{\mathrm{cr}}[\rho](q)=\zeta \int_{\mathbb{R}} \partial_{q} \tilde{G}\left(q, q^{\prime}\right) \rho\left(q^{\prime}\right) d q^{\prime}$. Here $\tilde{G}\left(q, q^{\prime}\right):=\left|q-q^{\prime}\right|$ is the $1 \mathrm{D}$ Green's function which fulfills $\partial_{q}^{2} \tilde{G}\left(q, q^{\prime}\right)=2 \delta\left(q-q^{\prime}\right)$. Therefore the integral kernel $G\left(q, q^{\prime}\right)$ for the CR beam-beam kick can be identified with $\partial_{q} \tilde{G}\left(q, q^{\prime}\right)=\operatorname{sgn}\left(q-q^{\prime}\right)$.

It follows immediately from the form of the kernel that the CR kick on a particle at $q$ is proportional to the number of particles of beam $*$ at a spatial position less than $q$ minus the number of particles at a spatial position larger than $q$. In particular, we find $\lim _{q \rightarrow \pm \infty} K_{\mathrm{cr}}[\rho](q)=\mp \zeta \neq 0$. This appears unphysical only at first sight. Since we assume flat beams and motion in the perpendicular plane, the charge distribution of the macroparticles of beam $*$ are actually represented by planes of constant planar density. Similar to the case of a capacitor with infinitely large plates, the field on a test particle due to each of the charge planes does not depend on the distance of the particle to the plane.

Moreover, the simple structure of the CR kernel $G$ allows the computation of the beam-beam kick on $N$ macroparticles exerted by an ensemble of $N^{*} \approx N$ particles, both located at arbitrary position in configuration space, with less than $O\left(N^{2}\right)$ operations. We can order the 
$N+N^{*}$ particles in sequence of increasing $q$. This can be done, for example, by the HEAPSORT algorithm [16], at an expense of $O(N \log N)$. Then one starts with the "leftmost" particle which receives a kick of $-\zeta$, the next particle receives a kick of $-\zeta$ if it belongs to the same beam as the leftmost particle or $-\zeta\left(1-\mathfrak{W}_{l}\right)$ if it belongs to the other beam, and so on. Here $\mathfrak{S}_{l}$ is the weight of the leftmost particle. This last step only has an operations count of $O(N)$ so that the total asymptotic order count of the algorithm is determined by the sorting and hence $O(N \log N)$.

With the Gaussian density $\rho\left(q ; \mu^{*}, \sigma^{*}\right):=$ $1 /\left(\sqrt{2 \pi} \sigma^{*}\right) \exp \left(-\frac{\left(q-\mu^{*}\right)^{2}}{2 \sigma^{* 2}}\right)$, we find

$$
\begin{aligned}
K_{\mathrm{cr}}[\rho](q) & =\zeta \int \operatorname{sgn}\left(q-q^{\prime}\right) \rho\left(q^{\prime} ; \mu^{*}, \sigma^{*}\right) d q^{\prime} \\
& =\zeta\left[2 \operatorname{erf}\left(\frac{q-\mu^{*}}{\sigma^{*}}\right)-1\right],
\end{aligned}
$$

where we define $\operatorname{erf}(q):=\int_{-\infty}^{q} \rho\left(q^{\prime} ; 0,1\right) d q^{\prime}, \quad \mu^{*}$ as the instantaneous distance of the centroid of the other beam from the common closed orbit, and $\sigma^{*}$ as the instantaneous rms width of the other beam. This formula describes the nonlinear beam-beam kick in the GSA and determines $\xi(\zeta)$ for initially Gaussian beams. From Eq. (5) and $\frac{d}{d q} K_{\mathrm{cr}}[\rho]=2 \zeta \rho\left(q ; \mu^{*}, \sigma^{*}\right)$, we find

$$
\xi_{\mathrm{cr}}:=\frac{\left.\beta_{0} \frac{d}{d q} K_{\mathrm{cr}}[\rho]\right|_{q=\mu^{*}=0}}{4 \pi}=(2 \pi)^{-3 / 2} \frac{\zeta \beta_{0}}{\sigma^{*}}
$$

\section{B. The axially symmetric force}

In the axially symmetric case the kick is given by Eq. (28). Assuming infinitely long axially symmetric bunches in both beams, i.e., in cylindrical coordinates with origin at the centroid $(\langle(x, y)\rangle)$, the spatial density of each beam fulfills $\partial_{z} \rho=\partial_{\phi} \rho=0$. Note that, although we treat the head-on case only, the instantaneous centroids of the beams are not required to be on the common closed orbit nor are they required to be identical for both beams. The beam-beam force after a Lorentz boost into the rest frame of the source beam is then given by the electric field $\vec{E}=: E(r) \hat{r}$, where $\hat{r}$ is the radial unit vector and $r=\sqrt{x^{2}+y^{2}}$. Gauss' law yields, for any cylinder $Z$ with radius $r$ and length $\Delta_{l}$,

$$
\begin{aligned}
\int_{\partial Z} \vec{E} \cdot \hat{r} d S & =\Delta_{l} 2 \pi r E(r)=\int_{Z} \rho(r) d V \\
& =\Delta_{l} 2 \pi \int_{0}^{r} \rho\left(r^{\prime}\right) r^{\prime} d r^{\prime},
\end{aligned}
$$

and thus $E(r)=1 / r \int_{0}^{r} \rho\left(r^{\prime}\right) r^{\prime} d r^{\prime}$. Undoing the Lorentz boost and absorbing all constants into $\zeta$ we find Eq. (28). We can then, under the premise that the beam stays round, choose an arbitrary transverse direction for $\hat{r}$. This procedure is applied usually to the strong source beam in weak-strong simulations.
Unfortunately, under the full Vlasov evolution the assumption of axial symmetry is not self-consistent. For a 1D model this means that the consistency constraint that the density is left-right symmetric about the centroid $\left[\rho_{\theta}\left(\langle q\rangle_{\theta}+q^{\prime}\right)=\rho_{\theta}\left(\langle q\rangle_{\theta}-q^{\prime}\right) \forall q^{\prime}\right]$ is in general not fulfilled for all $\theta$. In particular, the above constraint implies that all odd order centered moments $\left\langle(q-\langle q\rangle)^{2 n+1}\right\rangle_{\theta}$ vanish identically. In order to obtain a self-consistent $1 \mathrm{D}$ approximation of a round beam we modify the beam-beam kick to use the symmetrized density $\frac{1}{2}\left[\rho_{\theta}\left(\langle q\rangle_{\theta}+q^{\prime}\right)+\right.$ $\left.\rho_{\theta}\left(\langle q\rangle_{\theta}-q^{\prime}\right)\right]$. This is equivalent to taking the average of $\rho_{\theta}\left(\langle q\rangle_{\theta}+x\right)$ at $+q^{\prime}$ and at $-q^{\prime}$. Thus we can write the beam-beam kick

$$
\begin{aligned}
K_{\mathrm{as}}\left[\rho_{\theta_{c}^{-}}^{*}\right](q)= & \frac{1}{q-\langle q\rangle_{\theta_{c}^{-}}^{*}} \\
& \times \int_{-\left|q-\langle q\rangle_{\theta_{c}^{-}}^{*}\right|}^{+\left|q-\langle q\rangle_{\theta_{c}^{-}}^{*}\right|} \rho^{*}\left(\langle q\rangle_{\theta_{c}^{-}}^{*}+q^{\prime}\right)\left|q^{\prime}\right| d q^{\prime} .
\end{aligned}
$$

One can easily see that for any $\rho$ such that $\langle|q|\rangle$ exists, the limit $q \rightarrow \pm \infty$ of $K_{\text {as }}[\rho](q)$ vanishes at least linearly with $1 /\left|q-\langle q\rangle^{*}\right|$.

The kernel for the AS beam-beam kick is

$$
\begin{aligned}
G\left(q, q^{\prime}\right) & =\frac{1}{q-\langle q\rangle_{\theta_{c}^{-}}^{*}} \chi\left(\langle q\rangle_{\theta_{c}^{-}}^{*}, q, q^{\prime}\right)\left|q^{\prime}-\langle q\rangle_{\theta_{c}^{-}}^{*}\right|, \\
\chi\left(\mu, q, q^{\prime}\right) & := \begin{cases}1, & \left|q^{\prime}-\mu\right|<|q-\mu|, \\
0, & \left|q^{\prime}-\mu\right| \geq|q-\mu| .\end{cases}
\end{aligned}
$$

Note that here the kernel itself depends on the first moment of $\rho_{\theta_{c}^{-}}^{*}$.

With a Gaussian density $\rho\left(q^{\prime} ; \mu^{*}, \sigma^{*}\right)$ as in Sec. III A, the beam-beam kick is

$$
K_{\mathrm{as}}[\rho](q)=\sqrt{\frac{2}{\pi}} \zeta \frac{\sigma^{*}}{q-\mu^{*}}\left(1-e^{-\left[\left(q-\mu^{*}\right)^{2} / 2 \sigma^{* 2}\right]}\right) .
$$

And, analogously to Sec. III A,

$$
\xi_{\text {as }}=\frac{\zeta}{2}(2 \pi)^{-3 / 2} \frac{\beta_{0}}{\sigma^{*}} .
$$

As yet, no algorithm for computing the exact AS beambeam kick (34) at a lower order count than $O\left(N^{2}\right)$ has been found. However, the preliminary numerical results of this study, to be presented in Sec. V, seem to indicate that once the axial symmetry is put in by hand, the additional assumption of a Gaussian source does not change the moment calculation significantly. This is consistent with Yokoya's observation [15] that the major flaw of the GSA is its inability to represent the (acquired) skewness of the density. The symmetrization in Eq. (34) already removes the skewness from the actual density even before a test density is introduced. 


\section{The Yokoya-Koiso-Zenkevich force}

In the Yokoya-Koiso-Zenkevich case the kick is given by Eq. (29). The Yokoya-Koiso-Zenkevich force was derived [3] from the limit $\sigma_{x} \gg \sigma_{y}$ in the integral that solves the 2D Poisson problem

$$
\begin{aligned}
K_{\mathrm{yo}}[\rho](x)= & \zeta \lim _{\sigma_{y} / \sigma_{x} \rightarrow 0} \\
& \times \int_{\mathbb{R}^{2}} \partial_{x} \tilde{G}\left(x, y, x^{\prime}, y^{\prime}\right) \rho\left(x^{\prime}, y^{\prime}\right) d x^{\prime} d y^{\prime},
\end{aligned}
$$

where $\tilde{G}\left(x, y, x^{\prime}, y^{\prime}\right)$ is the 2D Green's function $\log \left(\sqrt{\left(x-x^{\prime}\right)^{2}+\left(y-y^{\prime}\right)^{2}}\right)$. Its kernel is singular and therefore a more careful treatment of the Yokoya-Koiso-Zenkevich force in the context of WMPT is needed. We do this in Appendix A and note here only that the Cauchy principle value is not very well represented in WMPT unless the GSA is used.

It can be shown with some algebra (see Appendix B) that with a Gaussian density $\rho\left(x ; \mu^{*}, \sigma^{*}\right)$, as in Secs. III A and III B, the beam-beam kick is

$$
\begin{aligned}
K_{\mathrm{yo}}[\rho](x) & =\sqrt{\frac{\pi}{2}} \frac{\zeta}{\sigma^{*}} \Im W\left(\frac{x-\mu^{*}}{\sqrt{2} \sigma^{*}}\right), \\
W(z) & :=e^{-z^{2}}\left(1+\frac{2 i}{\sqrt{\pi}} \int_{0}^{z} e^{t^{2}} d t\right), \quad z \in \mathbb{C} .
\end{aligned}
$$

Here $W$ is the complex "error" function [17] which is implemented, for example, in the CERNLIB [18]. And, analogously to Secs. III A and III B,

$$
\xi_{\text {yo }}=\frac{\zeta}{4 \pi} \frac{\beta_{0}}{\sigma^{* 2}} .
$$

Note that in the YO limit the beam width $\sigma^{*}$ appears squared in the denominator in contrast to the $\mathrm{CR}$ and the AS limit where it only appears linearly in the denominator. This is because the kernel has the dimension of $q^{-1}$ in the YO case and is dimensionless in the CR and the AS cases.

In the last two sections we will discuss only the beambeam interaction in the $p p$ case. Therefore, $\zeta$ and thus $\xi_{\mathrm{cr}}, \xi_{\mathrm{as}}$, and $\xi_{\text {yo }}$ are negative by definition. Nevertheless, we will for convenience redefine $\xi$ in the $p p$ case via $Q=Q_{0}-\xi+O\left(\kappa^{2} \beta^{2}\right)$, i.e., $\xi \rightarrow-\xi$.

\section{ACCURACY CONSIDERATIONS FOR WMPT}

The primary error in the method comes from the computation of the kicks, as discussed in Sec. II B. As mentioned there, we are looking at simple models to try to obtain a feel for the optimal $\Delta$ given $\Theta$. Wollman [11-13] has given a convergence proof for a 1D model used in plasma physics to describe Coulomb-interacting electrons with a fixed ion background. Here we present some preliminary simulations which give us some confidence in the method before proceeding to the simulations of the $\pi$ and the $\sigma$ modes.
We first discuss the choice of the mesh size. We then discuss the evolution of the first four moments of $q$ in the case where the two beams are identical and the initial distribution is an equilibrium of the linearized beam-beam force. Finally, we discuss in Figs. 3 and 4 the particle distribution after $2^{17}=131072$ turns in a special case.

The discretization scale $\Delta$ is (in principle for $p$ and $q$ independently) given by the initial mesh size divided by the number of macroparticles per dimension. Here and in Sec. V we chose normalized coordinates $\left(\beta_{0}=1 \Rightarrow\right.$ $\sigma_{q, 0}=\sigma_{p, 0}=: \sigma_{0}$ ), a square mesh from $-5 \sigma_{0}$ to $+5 \sigma_{0}$, and between $51\left(\Delta=0.2 \sigma_{0}\right)$ and $401\left(\Delta=0.025 \sigma_{0}\right)$ macroparticles per phase space dimension, initially uniformly distributed on a square mesh for both beams. We cannot expect dynamics on a spatial scale that are much smaller than the discretization scale $\Delta$ of the initial mesh to be visible during our simulations. In fact, we should consider every effect that appears at a scale $\ll \Delta$, e.g., small amplitude fluctuations of the position of the beam centroid or of the square root of the beam emittance, as an artifact of the unavoidable discretization noise.

In the case of the linearized beam-beam force there are stationary densities in which each beam has the same density. Given a density such that $\langle q\rangle=0$ there is a $\kappa$ defined by Eq. (5). This $\kappa$ gives the perturbed betatron ellipses defined by Eq. (8). It can be shown that there are densities whose equal density contours match the associated betatron ellipses. A special example for such a stationary solution of the linearized beam-beam force is the double Gaussian

$\psi(\vec{z})=\frac{1}{2 \pi \sigma^{2}} e^{-(1 / 2) \vec{z}^{T} \underline{C}^{-1} \vec{z}}, \quad \underline{C}:=\sigma^{2}\left(\begin{array}{cc}\beta & -\alpha \\ -\alpha & \gamma\end{array}\right)$,

where the perturbed Courant-Snyder functions are given by Eq. (8). This is discussed in some detail in [10]. It is also announced there that in the corresponding VlasovFokker-Planck system (i.e., with the addition of damping and diffusion due to, for example, radiation) there exists a unique stationary solution. Numerical simulations suggest that this solution is stable for small current. It is not yet known if equilibria exist in the radiation free case or if the equilibria are stable in the radiation free case with a linearized force. In $[19,20]$ we have shown that, for sufficiently small $\xi$, densities that depend only on the orbital action are quasiequilibria of the CR force in the sense that they only change $O(\xi)$ on time scales $O(1 / \xi)$. The results of WMPT below are consistent with an approximately stable density over $2^{17}$ turns. This is also consistent with the behavior of actual beams in colliders which normally show only a slow emittance growth.

In the CR case, which is so far the only one where WMPT can be done at less than $O\left(N^{2}\right)$ without GSA, we studied the evolutions of a distribution that is stationary under the linearized beam-beam force. Using Eq. (8) with $Q_{0}=\sqrt{5}-2, \xi=3 \times 10^{-3}, \beta_{0}=1$, and $\alpha_{0}=0$, we 
find $\beta \approx 1.0018, \alpha \approx-0.0188$, and $\gamma \approx 0.9985$ just after the kick. Note that this is still fairly close to the invariant circles of the unperturbed motion. We chose identical double Gaussians for each beam, given by Eq. (41). We simulate $2^{17}$ turns with and without the Gaussian source approximation with $n=51,101,201,401$ and with $n=$ 51, 101, and 201 macroparticles per phase space dimension, respectively.

Figure 2 shows the evolution of the centroid amplitude $\langle q\rangle_{m 2 \pi}$ as a function of the number of turns $m$. The vertical scale is the logarithm to base 10 of $\langle q\rangle_{m 2 \pi}$ in units of $\sigma_{0}$, and the horizontal scale is $m$. All scatter plots start at around $10^{-16}$ (double precision). With the "exact" computation of $K_{\mathrm{cr}}\left[\rho^{*}\right]$ (Fig. 2, left) the centroid amplitude immediately jumps to about $10^{-5}$ and then exponentially (linearly in the logarithmic scale) grows until a saturation limit is reached. With the additional smoothing of the GSA (Fig. 2, right) the centroid amplitude grows exponentially from $10^{-16}$ to a saturation limit.

The decrease in the saturation level with $n$ seems to be significant, and the saturation level itself is consistent with the size of $\Delta$ under the assumption of a quasistationary state with $\langle q\rangle=0$. The logarithmic slope of the envelope seems to be independent of $n$ in the exact case, whereas in the case of the GSA the logarithmic slope seems to be roughly proportional to $n^{-3 / 4}$. We do not understand this slope nor the jump from $10^{-16}$ to $10^{-5}$ in the exact case.

The second-order centered moments and therefore the beam emittance

$$
\epsilon:=\sqrt{\left\langle(q-\langle q\rangle)^{2}\right\rangle\left\langle(p-\langle p\rangle)^{2}\right\rangle-\langle(q-\langle q\rangle)(p-\langle p\rangle)\rangle^{2}}
$$

stay constant on the $0.1 \%-5 \%$ level (monotonically improving with decreasing discretization scale) in all simulations starting with a stationary Gaussian density of the linearized motion. The centered fourth-order moments are consistent with the assumption of a Gaussian beam (e.g., $\left\langle\left(q-\mu_{q}\right)^{4}\right\rangle_{\theta}=3\left(\left\langle\left(q-\mu_{q}\right)^{2}\right\rangle_{\theta}\right)^{2}$ with $\left.\mu_{q}=\langle q\rangle_{\theta}\right)$ also on the level of a couple of percent.

The centered third-order moments oscillate around 0 . Similar to the first-order moments, their oscillation amplitude grows exponentially with time until it saturates. The saturation level decreases with decreasing $\Delta$, but at a higher value than the level for $\langle q\rangle$. Note that, for a Gaussian distribution, as for any distribution whose density is even around its mean, all odd centered moments vanish identically. Thus the relatively high saturation level of the third-order moments suggests that the GSA should be revised and a different test density which allows odd centered moments should be used in Eq. (25).

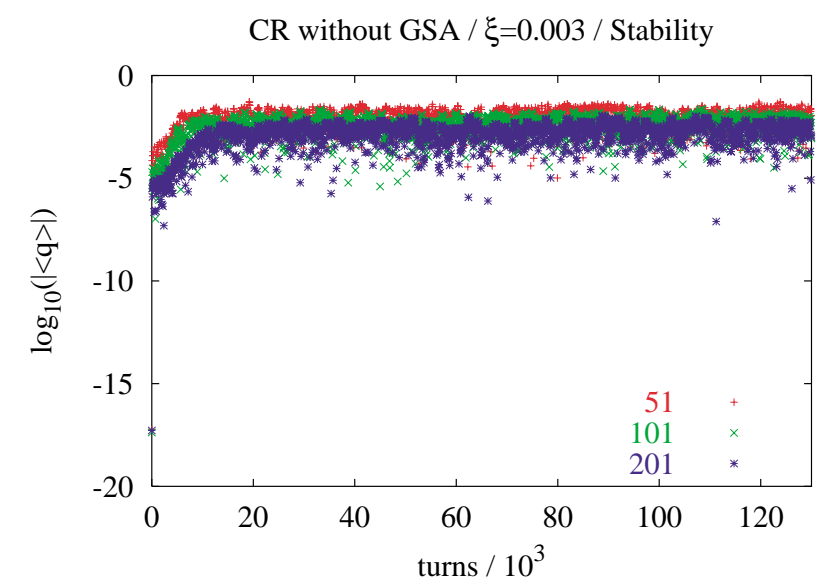

In all simulations discussed so far the agreement between $\left\langle q^{v} p^{w}\right\rangle_{\theta}$ and $\left\langle q^{v} p^{w}\right\rangle_{\theta}^{*}$ was better then $10^{-4}$, which is not surprising for an evolution equation being symmetric under $\psi \leftrightarrow \psi^{*}$ and identical initial conditions for both beams.

Similar simulations exist for the AS and the YO beambeam limits, both in the Gaussian source approximation, and they show qualitatively the same stability properties as the case of the CR interaction. Moreover, they give quantitatively similar results concerning saturation amplitudes and logarithmic slopes. The approximate saturation levels of the fluctuations of the first and third moments, as well as the fluctuations in the beam emittance, are shown in Table I.

The numerical simulations shown in Fig. 2 and Table I not only give some confidence in WMPT, but also seem to indicate the existence of at least quasistationary phase space densities under the full nonlinear collective beambeam interaction in $1 \mathrm{D}$.

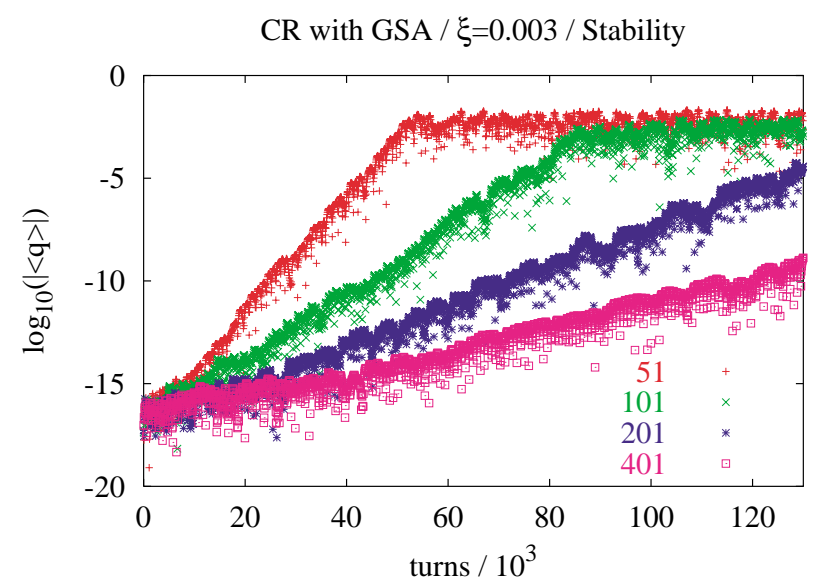

FIG. 2. (Color) The centroid position $\langle q\rangle$ of the linearly matched phase space distribution with WMPT for the CR beam-beam force. $\langle q\rangle$ is normalized by $\sigma_{0}$. Only each 64th turn is actually printed. Left: "exact" $[O(N \log N)]$ computation of the beam-beam kick $(n=51,101$, and 201). Right: Gaussian source approximation $(n=51,101,201$ and 401$)$. 
TABLE I. The approximate bounds of $\langle q\rangle$ and $\left\langle(q-\langle q\rangle)^{3}\right\rangle$ and the approximate maximum deviation of the emittance from $\sigma_{0}^{2}$ for the four model forces under numerical study. $\Delta$ is the grid spacing.

\begin{tabular}{ccccc}
\hline \hline Force & $\Delta / \sigma_{0}$ & $\max |\langle q\rangle| / \sigma_{0}$ & $\max \left|1-\epsilon / \sigma_{0}^{2}\right|$ & $\max \left|\left\langle(q-\langle q\rangle)^{3}\right\rangle / \sigma_{0}^{3}\right|$ \\
\hline CR & 0.200 & 0.06 & 0.05 & 0.2 \\
& 1.000 & 0.025 & 0.015 & 0.1 \\
& 0.050 & 0.01 & 0.001 & 0.05 \\
CR/GSA & 0.200 & 0.025 & 0.004 & 0.2 \\
& 0.100 & 0.008 & 0.0008 & 0.1 \\
& 0.050 & & No saturation in $2^{17}$ turns \\
AS/GSA & 0.025 & & & \\
& 0.200 & 0.05 & 0.0025 & 0.25 \\
& 0.100 & 0.02 & 0.001 & 0.12 \\
YO/GSA & 0.050 & 0.007 & 0.0005 & 0.04 \\
& 0.200 & 0.06 & 0.01 & 0.4 \\
& 0.100 & 0.015 & 0.002 & 0.1 \\
\hline \hline
\end{tabular}

Since in the WMPT approach the phase space integrals in the expectation values $\langle f\rangle_{\theta}$ and in the beam-beam kicks $K\left[\rho_{\theta_{c}^{-}}^{*}\right]$ are approximated by sums over the trajectories $\vec{\eta}_{i j}$ and $\vec{\eta}_{i j}^{*}$, respectively, it has to be checked whether or not the effective distribution of the trajectories leads to a sufficiently slowly varying coarse grained density in the sense of Eq. (20). Although "spikes" and "holes" in the coarse grained density might as well be of physical origin, one might expect that the numerical accuracy of the numerical representation of the phase space integral suffers if the coarse grained density is too rapidly varying.

Figure 3 (left) shows a scatter plot of all the trajectories of one beam after $2^{17}$ turns. The initial mesh was set up identically to the simulations for Fig. 2 with $201 \times 201$ macroparticles per beam, but the beams were initially exactly round (in the normalized coordinates) and the "other" beam (not shown) had an initial coherent betatron amplitude of $0.1 \sigma_{0}$. Again the beam-beam tune shift parameter was chosen to be $3 \times 10^{-3}$. The unperturbed tunes were $Q_{0}^{*}=\sqrt{5}-2$ and $Q_{0}=Q_{0}^{*}-5 \times 10^{-6}$. Therefore the

CR without GSA $/ \xi=0.003 /$ ScatterPl.

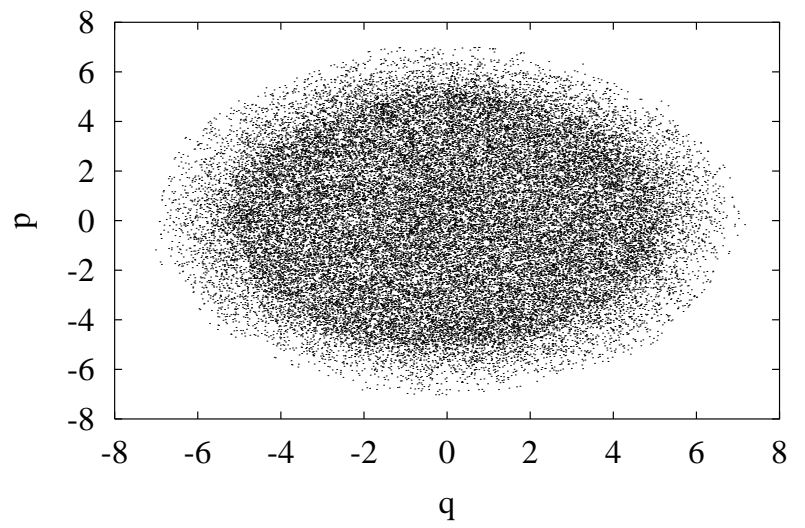

beams are expected to show persistent collective modes $[2-6,21]$ and, indeed, in Sec. V the existence of a $\pi$ and a $\sigma$ mode will be demonstrated. It has to be noted that in all simulations performed for this study thus far, the unperturbed tunes were chosen far away from all lower order resonances of the single particle motion.

The scatter plot in Fig. 3 (left) shows the distribution of the trajectories in the phase plane after $2^{17}$ turns. Note that the initial mesh was square from -5 to $+5 \sigma_{0}$ in both phase space dimensions. Thus the particles with the largest initial incoherent betatron amplitude are at a distance of $\left(\sqrt{5^{2}+5^{2}} \approx 7\right) \sigma_{0}$ from the center. The distribution appears uniform except for a halolike ring of reduced point density. The appearance of this halolike ring from about $5 \sigma_{0}$ to about $7 \sigma_{0}$ is most likely an artifact of the square initial mesh. We thus conclude that the macroparticles remain uniformly distributed in a coarse grained sense.

Figure 3 (right) shows a 3D scatter plot of the macroparticle weights $\mathfrak{S}_{i j}=\psi_{0}\left(\vec{z}_{i j}\right) w_{i j}$ over the actual position of the trajectory $\vec{\eta}_{i j}(\theta)$ after $2^{17}=131072$ turns. Since in

\section{CR without GSA / $\xi=0.003 /$ ScatterPl.}

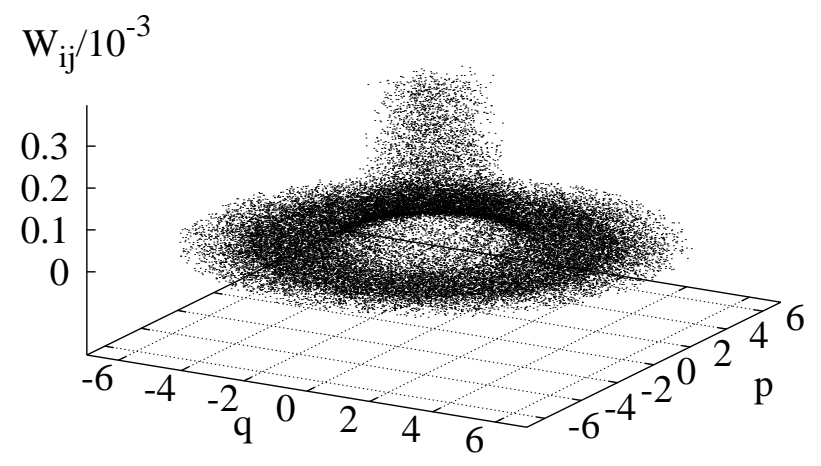

FIG. 3. Scatter plots of $\vec{\eta}_{i j}(\theta)$ (left) and $\left(\vec{\eta}_{i j}(\theta), \mathfrak{W}_{i j}\right)$ after $2^{17}$ turns. $q$ and $p$ are normalized by $\sigma_{0}$. 
CR without GSA / $\xi=0.003 /$ MeshProj.

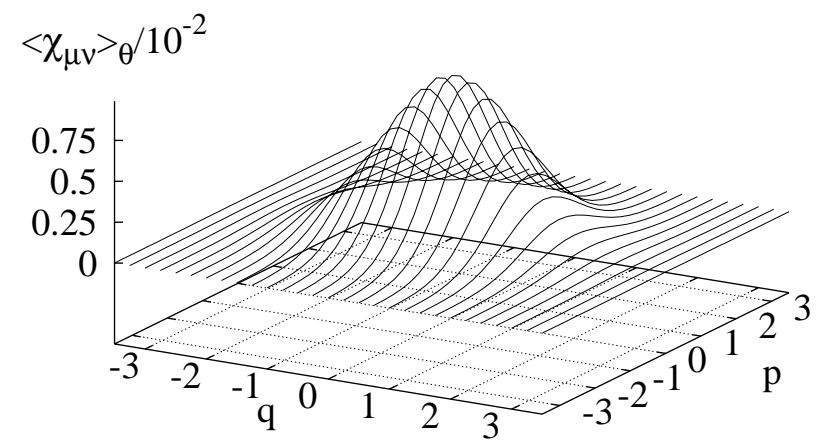

CR without GSA / $\xi=0.003 /$ MeshProj.

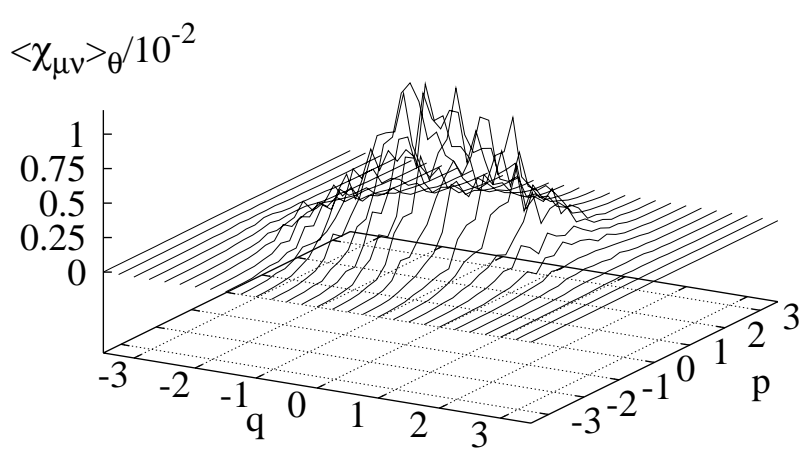

FIG. 4. Mesh projections $\left\langle\chi_{\mu \nu}\right\rangle_{\theta}$ after 0 turns (= initial data) (left) and after $2^{17}$ turns (right). The mesh that $\psi_{\theta}$ was projected on was in both cases given by $41 \times 41$ mesh points from -5 to $+5 \sigma_{0}$ and the initial mesh of the macroparticles had $201 \times 201$ points in the same range. $q$ and $p$ are normalized by $\sigma_{0}$. To emphasize the shape of the core, only the range from -3.5 to $+3.5 \sigma_{0}$ is shown.

the current implementation of the algorithm $w_{i j}=w=$ const, and since the initial density was a round centered Gaussian, the vertical coordinate of each point is a measure for the initial distance from the origin: $\sqrt{q_{i}^{2}+p_{j}^{2}}=$ $\sqrt{-2 \ln \left[2 \pi \psi_{0}\left(\vec{z}_{i j}\right)\right]}$. Obviously the majority of particles that initially belonged to the core stay relatively close to the core. Otherwise the scatter plot would look less similar to a "bell" and more similar to a uniform cloud.

Figure 4 shows projections of $\psi_{\theta}$ [Eq. (20)] on a mesh of $41 \times 41$ mesh points for the initial Gaussian (left) and after $2^{17}$ turns (right). The parameters of the simulation were the same as for Fig. 3. A scatter plot of the initial data would of course coincide with the surface of Fig. 4 (left). But after $2^{17}$ turns the scatter plot Fig. 3 (right) is a diffuse bell-shaped cloud and the coarse grained mesh projection gives an indication of the actual density. The mesh projection of Fig. 4 (right) looks a little jagged. This is due in part to the particular choice of the partition of unity in this mesh projection. It is also due in part to the dynamics that can bring two initially separated particles, with significantly different densities, close together. However, the mesh projection has a well-defined core, and each of the core bins has a significant amount of probability assigned to it. Moreover, the projected density at the edge of the core decreases relatively smoothly, and there are no islands outside the core. The coarse grained density as obtained by $2^{17}$ turns of WMPT therefore has a slowly varying component (the bell) and a rapidly varying component (the peaks and valleys). In phase space averages the slowly varying part will be represented with relative high accuracy and the rapidly varying part, which contributes only little to the average, will be represented with relatively low accuracy.

Since WMPT is designed to compute integrals including the density rather than computing the density itself, we have gained confidence that the "smoothness" observed in the presented example, together with the apparent convergence in $\Delta$ observed in Table I and Fig. 2, seems sufficient to provide a relatively accurate time evolution of the low order moments on scales well above the discretization scale and for a finite number of turns.

\section{SIMULATIONS}

One key task of this stage of the study was to identify the $\pi$ and $\sigma$ modes for the three different 1D limits of the beam-beam force in head-on collisions and to discuss their dependence on the difference of the unperturbed tunes $\Delta Q:=Q_{0}-Q_{0}^{*}$ and $\Delta \xi:=\xi-\xi^{*}$. Moreover, the onset of Landau damping for $\Delta Q>\xi$ should be observable.

We performed a large number of simulation runs with different parameter sets, but only a fraction of them can be presented here.

For comparison we will use a trivial extension of the rigid bunch model described in [21]. Under the assumption of rigid bunches and a linearized beam-beam force, the motion of the beam centroids $X:=\langle q\rangle$ and $X^{*}:=\langle q\rangle^{*}$ is equivalent to a system of two coupled linear oscillators,

$$
\begin{gathered}
\frac{d^{2}}{d \theta^{2}} X+\left(Q_{0}^{2}+Q_{0} \xi\right) X-Q_{0} \xi X^{*}=0, \\
\frac{d^{2}}{d \theta^{2}} X^{*}+\left(Q_{0}^{* 2}+Q_{0}^{*} \xi^{*}\right) X^{*}-Q_{0}^{*} \xi^{*} X=0 .
\end{gathered}
$$

The eigentunes $Q^{+}$and $Q^{-}$are easily found to be

$$
\begin{aligned}
\left(Q^{ \pm}\right)^{2}=\frac{1}{2}[ & Q_{0}^{2}+Q_{0}^{* 2}+Q_{0} \xi+Q_{0}^{*} \xi^{*} \\
& \left.\mp \operatorname{sgn}(\xi) \sqrt{\left(Q_{0}^{2}-Q_{0}^{* 2}\right)^{2}+\left(Q_{0} \xi+Q_{0}^{*} \xi^{*}\right)^{2}+2\left(Q_{0}^{2}-Q_{0}^{* 2}\right)\left(Q_{0} \xi-Q_{0} \xi^{*}\right)}\right] .
\end{aligned}
$$


In the case of $\xi^{*}=\xi$ this reproduces the result presented in [21], and for $Q_{0}^{*}=Q_{0}$ one easily finds that the eigentunes $Q^{+}$and $Q^{-}$depend only on $Q_{0}$ and the mean $\left(\xi+\xi^{*}\right) / 2$. Finally, for both $\xi^{*}=\xi$ and $Q_{0}^{*}=Q_{0}$, we have

$$
\left.\left(Q^{ \pm}\right)^{2}=Q_{0}^{2}+Q_{0} \xi \mp \operatorname{sgn}(\xi)\left|Q_{0} \xi\right| \Rightarrow \stackrel{Q^{+}}{Q^{-}}\right\} \stackrel{|\xi| \ll Q_{0}}{\longrightarrow}\left\{\begin{array}{c}
Q_{0} \\
Q_{0}+\xi
\end{array}\right.
$$

In this case, the eigenmodes for $Q^{+}$and $Q^{-}$correspond to the $\sigma$ mode $\left(X+X^{*}\right)$ and the $\pi$ mode $\left(X-X^{*}\right)$. It is not surprising that in this simple model the $\pi$ mode frequency for $|\xi| \ll Q_{0}$ does not contain the Yokoya factor $Y\left(Q_{\pi}=\right.$ $Q+Y \xi$ ) which was predicted to differ from one in [3] by means of the linear Vlasov theory. Note that for $Q_{0}^{*} \neq Q_{0}$ the $\pi$ mode and the $\sigma$ mode are not the eigenmodes even of the rigid bunch model.

In the following, we will discuss the spectra obtained by simulations using WMPT and the onset of Landau damping of the modes and compare the most prominent frequencies of the spectra with the eigentunes $Q^{+}$and $Q^{-}$of the rigid bunch model.

\section{A. The dependence of the $\pi$ and $\sigma$ modes on $\Delta Q$}

In this section we will present results of simulations in the three cases: CR without GSA in Fig. 5, AS with GSA in Fig. 6, and YO with GSA in Fig. 7. The parameter sets will be basically identical. In particular, the beam-beam tune shift parameter is the same $\left(|\xi|=3 \times 10^{-3}\right)$ for all simulations in this section. The figures are structured as follows: the first three plots are tune spectra, i.e., the modulus of the Fourier amplitude computed by a fast Fourier transform (FFT) based on $2^{17}$ turns, for the $\pi$ mode $\langle q\rangle-\langle q\rangle^{*}$ (light/green) and the $\sigma$ mode $\langle q\rangle+\langle q\rangle^{*}$ (dark/blue). The spectra are normalized so that the largest amplitude is 1 . The thick vertical (dark/red) lines (cursors) that appear in all spectra are markers, e.g., $Q_{l}, Q_{r}, Q_{l}-$ $|\xi|, Q_{r}-|\xi|, Q^{+}$, and $Q^{-}$. The initial conditions for the simulations used to compute the spectra are round Gaussians, i.e., Gaussians matched to the unperturbed optics in normalized coordinates, with $\sigma_{0}=1$ for both beams, one beam (clockwise, right, " $r$ ") being initially centered around the common closed orbit and the other beam (counterclockwise, left, "l") having an initial coherent betatron amplitude of $0.1 \sigma_{0}$. The unperturbed tune of beam (1) was always set to $Q_{l}:=\sqrt{5}-2 \approx 0.23606798$ and the unperturbed tune of beam (r) was set to $Q_{r}:=Q_{l}-\Delta Q$, where $\Delta Q=5 \times 10^{-6}, 1.5 \times 10^{-3}$, or $6 \times 10^{-3}$ for the top left, top right, and bottom left plots, respectively. The only exception is the YO case (Fig. 7) where, because of reasons to be explained later, the top right plot has a $\Delta Q$ of $0.5 \times 10^{-3}$ instead of $1.5 \times 10^{-3}$. In all cases, the bottom right plot shows the time evolution of $\left\langle q_{l}\right\rangle$ (red crosses) and the beam emittance (light/green line) of beam (1) for the first $10^{4}$ turns and for $\Delta Q=6 \times 10^{-3}$. There the initial coherent betatron amplitude of beam (l) was $1.0 \sigma_{0}$.

The viewpoint for all simulations is $\theta_{c}^{+}$, i.e., the position directly after the IP, and the initial mesh has $201 \times 201$ points uniformly distributed on a rectangle in phase space from -5 to $+5 \sigma_{0}$.

Since the CR limit is the only one where a reasonably fast $[O(N \log N)]$ algorithm for the computation of the beam-beam kick without the additional simplification of the Gaussian source approximation has been found, Fig. 5 is the only figure that shows the results of simulations with the exact WMPT without GSA. The main and clearly visible features in Fig. 5 (top left) are the $\pi$ and $\sigma$ modes and the noisy continuum. The continuum originates from the single particle motion. It reflects the incoherent tune spread and falls off sharply at $Q_{l}-|\xi|$. In addition, it goes strongly to zero as the tune $Q \rightarrow Q_{l}$. In the weak-strong approximation, the weak beam has an amplitude dependent tune spread from $Q_{l}$ at infinitely large amplitude to $Q_{l}-|\xi|$ at zero amplitude. Since the initial phase space mesh has a cutoff at $\pm 5 \sigma_{0}$, and since the outermost macroparticles carry a basically vanishing weight, the amplitude of the continuum decreases strongly as the frequency parameter $Q$ approaches $Q_{l}$. The $\sigma$ mode (blue) has a sharp peak at $Q_{l}$ as expected by the theory $[2-5,21]$. The position of the peak amplitude of the $\pi$ mode (green) is at $0.231537 \approx Q_{l}-1.51|\xi|$. We cross checked the result with the PF method in $[19,22]$ and found the same Yokoya factor. Moreover, we derived an averaged Vlasov equation for the CR limit [20] and found exactly the Yokoya factor 1.513. We are quite surprised that the linearization of the averaged Vlasov equation yields essentially the same Yokoya factor as the fully nonlinear and completely self-consistent simulation. Note that the $\pi$ mode contains the noisy continuum whereas the $\sigma$ mode does not. This is a peculiarity of the CR force and we do not yet have an explanation.

Figure 5 (top right) shows the spectrum with $\Delta Q=$ $|\xi| / 2$. Very close to $Q^{+}$, at 0.23545 lies the most prominent peak of the $\sigma$ mode. Now both the $\sigma$ mode and the $\pi$ mode show a continuum that ends at $Q_{r}-|\xi|$. The largest peak of the $\pi$ mode is at a distance of $1.61|\xi|$ left of the largest peak of the $\sigma$ mode. We note that the $\pi$ and $\sigma$ modes are not the eigenmodes of the linearized motion when $\Delta Q \neq 0$. In fact, both modes have two prominent peaks at the same position. As we will see later, this is true for all three forces.

The envelopes of $\langle q\rangle_{\theta} \pm\langle q\rangle_{\theta}^{*}$ and the beam emittances, which are not shown here, are in both cases $(\Delta Q=5 \times$ $10^{-6}$ and $1.5 \times 10^{-3}$ ) basically constant and neither show significant damping of the centroid motion nor emittance blowup up to 130000 turns. This situation changes when $\Delta Q=2|\xi|$ (bottom two plots of Fig. 5). Note that both spectra have a strong peak close to $Q^{+}$. Moreover, the 

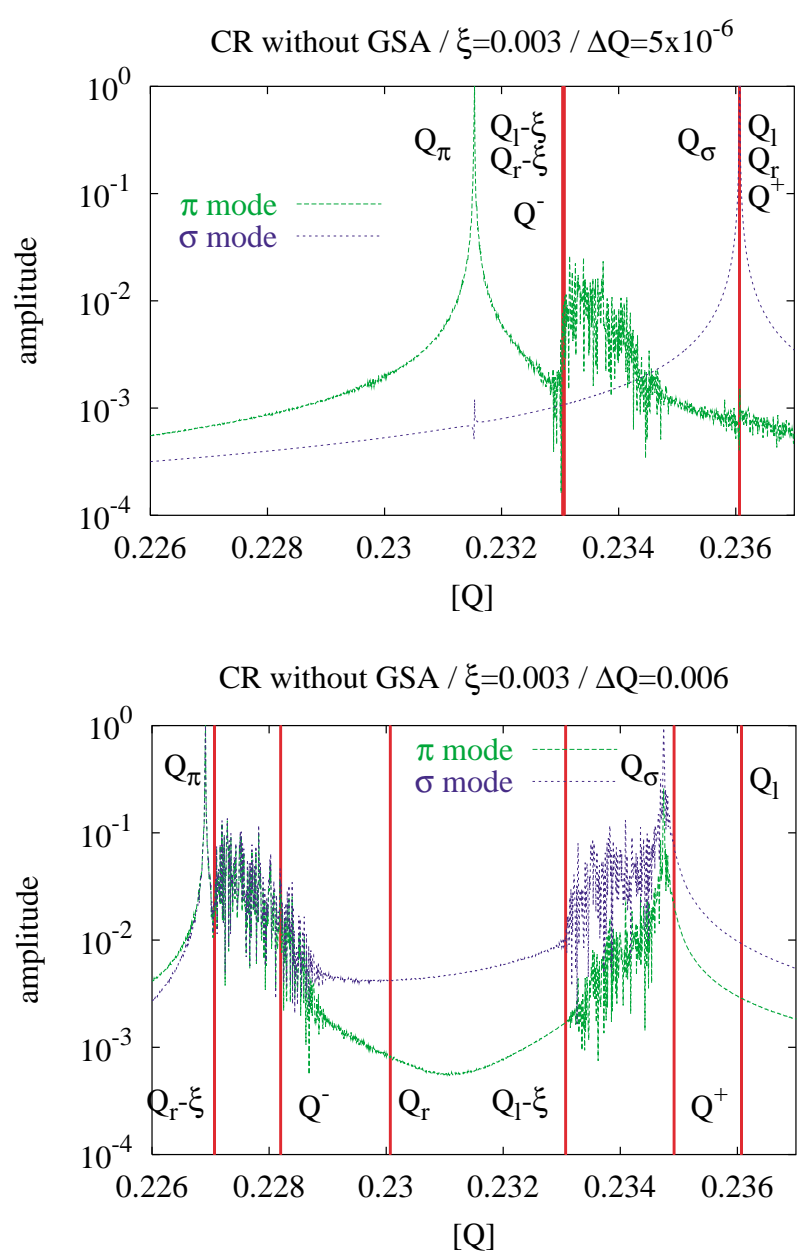
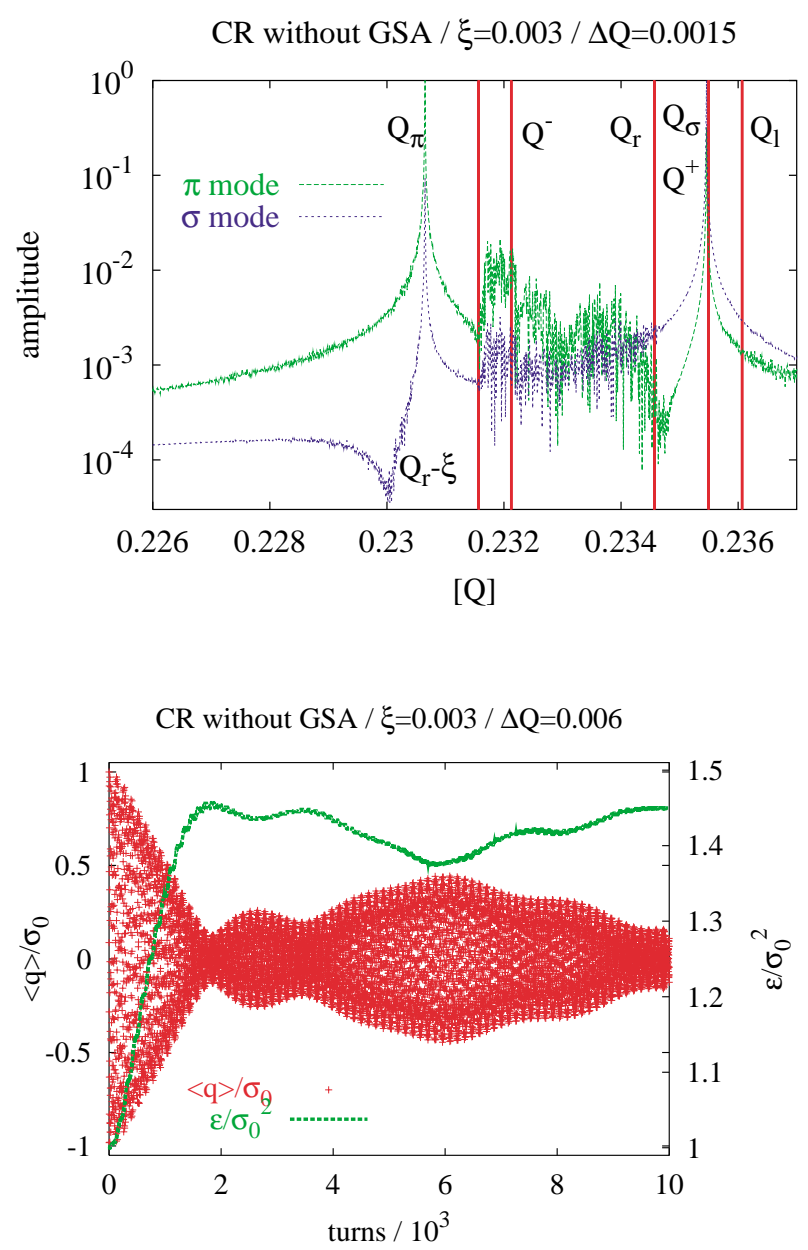

FIG. 5. (Color) Chao-Ruth limit: FFT analysis of the $\pi$ and $\sigma$ modes for $\Delta Q=5 \times 10^{-6}, 1.5 \times 10^{-3}$, and $6.0 \times 10^{-3}$ (clockwise top left to bottom left). Bottom right: time evolution of $\left\langle q_{l}\right\rangle$ and $\epsilon_{l}$ for the first 10000 turns.

continuum now has two disjoint parts. One continuum ranges from $Q_{l}-|\xi|$ to $Q^{+}$. The second part of the continuum ranges from about $Q_{r}-0.4|\xi|$ to $Q_{r}-|\xi|$, and slightly left of the continuum at 0.22691 lies the second strong peak of both the $\pi$ and the $\sigma$ modes. The two peaks are separated by $2.61|\xi|$. The strongest peak in both spectra is a little left of $Q_{r}-|\xi|$. We conclude that with this tune separation the characteristic separation of the main peak of both modes disappears. Nevertheless, with the $\mathrm{CR}$ force, even at $\Delta Q=2|\xi|$ there are still two distinct main peaks in the spectra. In Fig. 5 (bottom right) the amplitude (red points) of the centroid motion of the initially excited beam (r) decays with increasing revolution number on a scale larger than but comparable to $1 /|\xi|$. The emittance $\epsilon_{r}$ (light/green line) grows on the same time scale from initially $1 \sigma_{0}^{2}$ to between 1.4 and $1.5 \sigma_{0}^{2}$. These effects indicate the onset of the so-called Landau damping and are predicted by the linearized Vlasov theory [3-5] for $\Delta Q \gg|\xi|$. Comparing with Figs. 6 and 7 for the AS and the YO limits, respectively, we see that there the Landau-damping process leads to a much smaller stationary amplitude and a much more stable beam emittance.
Figure 6 shows a qualitatively similar situation for the AS interaction as in Fig. 5. Both plots on the top show clearly separated $\pi$ and $\sigma$ modes. With $\Delta Q=5 \times 10^{-6}$, i.e., $Q_{r} \approx Q_{l} \approx Q^{+}$, the $\sigma$ mode has its dominant peak at $Q_{l}$ and the single particle continuum, which is now visible in both modes, has a sharp boundary at $Q_{l}-|\xi| \approx Q^{-}$. The $\pi$ mode appears at about $Q_{l}-1.27|\xi|$. Relative to the CR case it is therefore shifted towards the $\sigma$ mode (and the continuum). In a similar simulation without GSA but with only $75 \times 75$ macroparticles, the $\pi$-mode peak which was much noisier was even shifted a little farther $\left(Q_{l}-1.25|\xi|\right)$ towards the $\sigma$ mode. This case can to some extent be compared to the predictions of the linearized Vlasov theory $[3,4]$, to the 2D simulations recently performed by Zorzano and Zimmermann [6], Herr et al. [23], and our own most recent 2D simulation [22]. The linearized Vlasov theory predicts the separation of $\pi$ and $\sigma$ modes of about $1.21|\xi|$. The 2D simulations, presented in [6], using an initially round beam represented by $10^{4}$ nonweighted macroparticles and employing the GSA, yield a separation of about $1.10|\xi|$. Their more recent result [23], using a completely self-consistent hybrid fast 

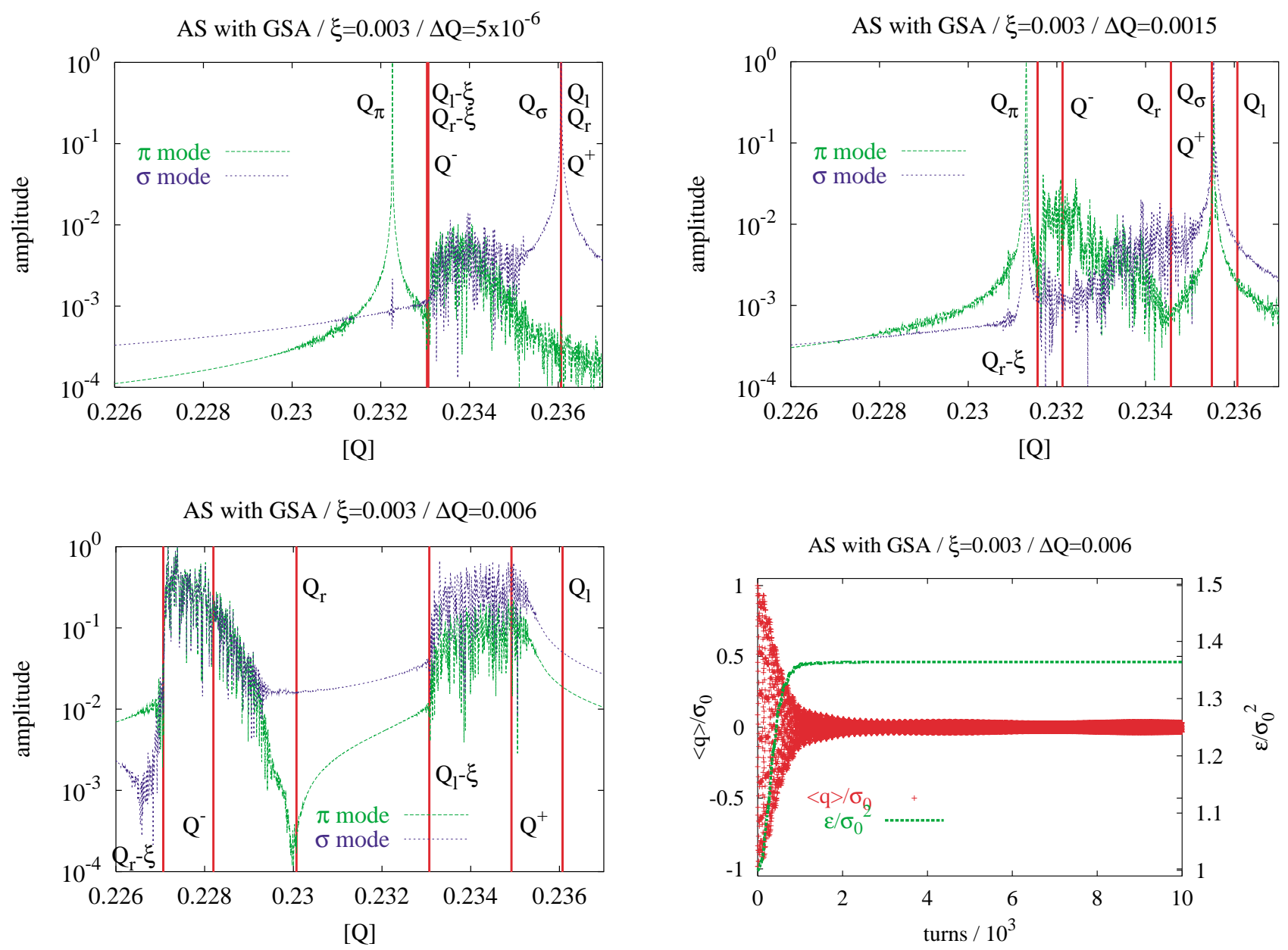

FIG. 6. (Color) Axially symmetric limit: FFT analysis of the $\pi$ and $\sigma$ modes for $\Delta Q=5 \times 10^{-6}, 1.5 \times 10^{-3}$, and $6.0 \times 10^{-3}$ (clockwise top left to bottom left). Bottom right: time evolution of $\left\langle q_{l}\right\rangle$ and $\epsilon_{l}$ for the first 10000 turns.

multipole method [24,25] but still using only $10^{4}$ nonweighted macroparticles per beam, reproduces the result of the linear Vlasov theory. We have also recently performed 2D simulations [22] using our own implementation of the hybrid fast multipole method and $45^{4} \approx 4.1 \times 10^{6}$ weighted macroparticles per beam and found a Yokoya factor of 1.28 for initially round beams. Our 1D result in the AS limit with $201^{2} \approx 4 \times 10^{4}$ weighted macroparticles and in GSA, namely $Y=1.27$, is surprisingly consistent with the fully self-consistent $2 \mathrm{D}$ simulations, provided the phase space is sampled in a comparable way. The analytic approach in [15] applied to a round beam in 2 degrees of freedom shows that, for infinitesimally small amplitudes, the GSA yields approximately half the value of $(Y-1)$ than the exact linearized Vlasov model. However, our simulations using the axially symmetric $1 \mathrm{D}$ model in the GSA and the self-consistent 2D model for initially round beams predict a Yokoya factor which is slightly above the result obtained from the linearized Vlasov theory. It is possible that, in contrast to the CR limit where we have found excellent agreement between theory and simulation, the round beam case is more sensitive to the nonlinear- ity of the beam-beam force introduced by the tails of the distribution. We note that in the conventional macroparticle approach the beam is represented by a Monte Carlo generated ensemble of macroparticles of equal weight. An initially Gaussian distribution therefore samples the core and the tail in different ways.

In the top right plot of Fig. 6 with $\Delta Q=1.5 \times 10^{-3}=$ $|\xi| / 2$ we see that the $\sigma$ mode has its major peak close to $Q^{+}$between $Q_{l}$ and $Q_{r}$. The position of the $\sigma$-mode peak is basically the same as compared to the CR interaction, but slightly shifted from $Q^{+}$towards the right. The $\pi$ mode appears at $Q_{\sigma}-1.41|\xi|$ but is already very close to the boundary of the single particle continuum at the leftmost cursor $Q_{r}-|\xi|$.

Figure 6 (bottom left) with $\Delta Q=2|\xi|$ does not show any prominent singular peak in either spectra. The absence of any dominant frequencies in the spectra shows that the coherent modes have disappeared. The plot at the bottom right of Fig. 6 supports this result. The amplitude of the initially excited beam is Landau damped to the discretization scale of the simulation, while the beam emittance is increased to about $1.37 \sigma_{0}^{2}$. 

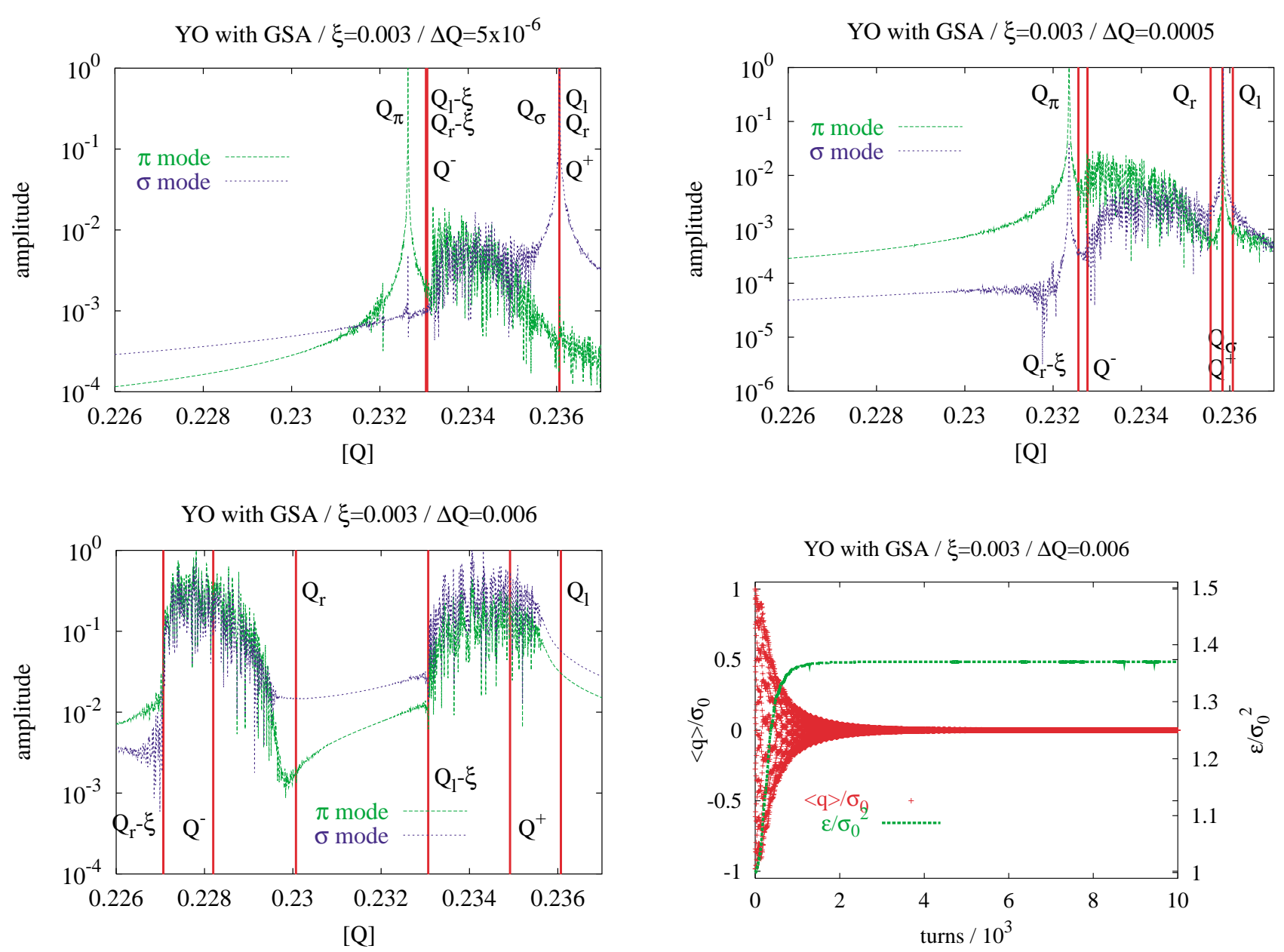

FIG. 7. (Color) Yokoya-Koiso-Zenkevich limit: FFT analysis of the $\pi$ and $\sigma$ modes for $\Delta Q=5 \times 10^{-6}, 0.5 \times 10^{-3}$, and $6.0 \times$ $10^{-3}$ (clockwise top left to bottom left). Bottom right: time evolution of $\left\langle q_{l}\right\rangle$ and $\epsilon_{l}$ for the first 10000 turns.

Figure 7 shows the equivalent simulations for the YO limit. As we can easily see in the top left plot, the $\pi$ mode is now separated by $1.14|\xi|$ from the $\sigma$ mode and thus even closer to the continuum. This again can be compared to the linear Vlasov theory applied in $[3,4,15]$ and the case of a flat beam (where "flat" means $\sigma_{x} / \sigma_{y}=16$ ) studied numerically in [6]. Yokoya and Koiso predict $1.33|\xi|$, and Zorzano and Zimmermann find 1.15. In [15] the Yokoya factor for the YO limit in the GSA is calculated as 1.148. Since the $\pi$ mode is so close to the continuum already with $\Delta Q \ll|\xi|$, it is not a surprise that with $\Delta Q=|\xi| / 2$ (not shown) the system is in a Landau-damped regime. In this case, the amplitude of the centroid motion is damped to some intermediate value and the spectra show no clear signature of well-defined $\pi$ and $\sigma$ modes. Therefore, Fig. 7 (top right) shows the spectra of the $\pi$ and $\sigma$ modes for $\Delta Q=0.5 \times 10^{-3}$ instead of $1.5 \times 10^{-3}$ as in the CR and the AS case. Here the $\pi$ mode is about $1.17|\xi|$ separated from the $\sigma$ mode. When $\Delta Q$ is increased to $6 \times 10^{-3}$ (bottom two plots), similar to the AS limit, the beam's centroid motions are more or less completely decoupled and the initial amplitude of beam (1) is quickly damped to a value below the discretization scale.

\section{B. The dependence of the $\pi$ and $\sigma$ modes on $\Delta \xi$}

It was suggested in [5] and numerically confirmed in [23] that the $\pi$ mode for round beams moves back into the continuum when the ratio $\xi / \xi^{*}$ is reduced to less than 0.6. In this section we briefly discuss our results in the three 1D cases. Figure 8 contains spectra of the $\pi$ (light/green) and $\sigma$ modes (dark/blue) for CR (top row), AS with GSA (center row), and YO with GSA (bottom row), and for $\xi_{r} / \xi_{l}=0.5$ (left column, upper two rows), $\xi_{r} / \xi_{l}=0.6$ (left column, bottom row), and 0.1667 (right column). Both unperturbed tunes were chosen to be identical $Q_{l}=Q_{r}=\sqrt{5}-2$, and the left beam had an initial coherent betatron amplitude of $0.1 \sigma_{0} . \xi_{l}=-0.003$ was kept constant, and $\xi_{r}$ was varied. The thick red cursors mark $Q^{+}=\sqrt{5}-2$ (right) and $Q^{-}$(left).

It is easy to see that in all six plots the $\sigma$ mode has its most pronounced peak at $Q^{+}$. Moreover, if 

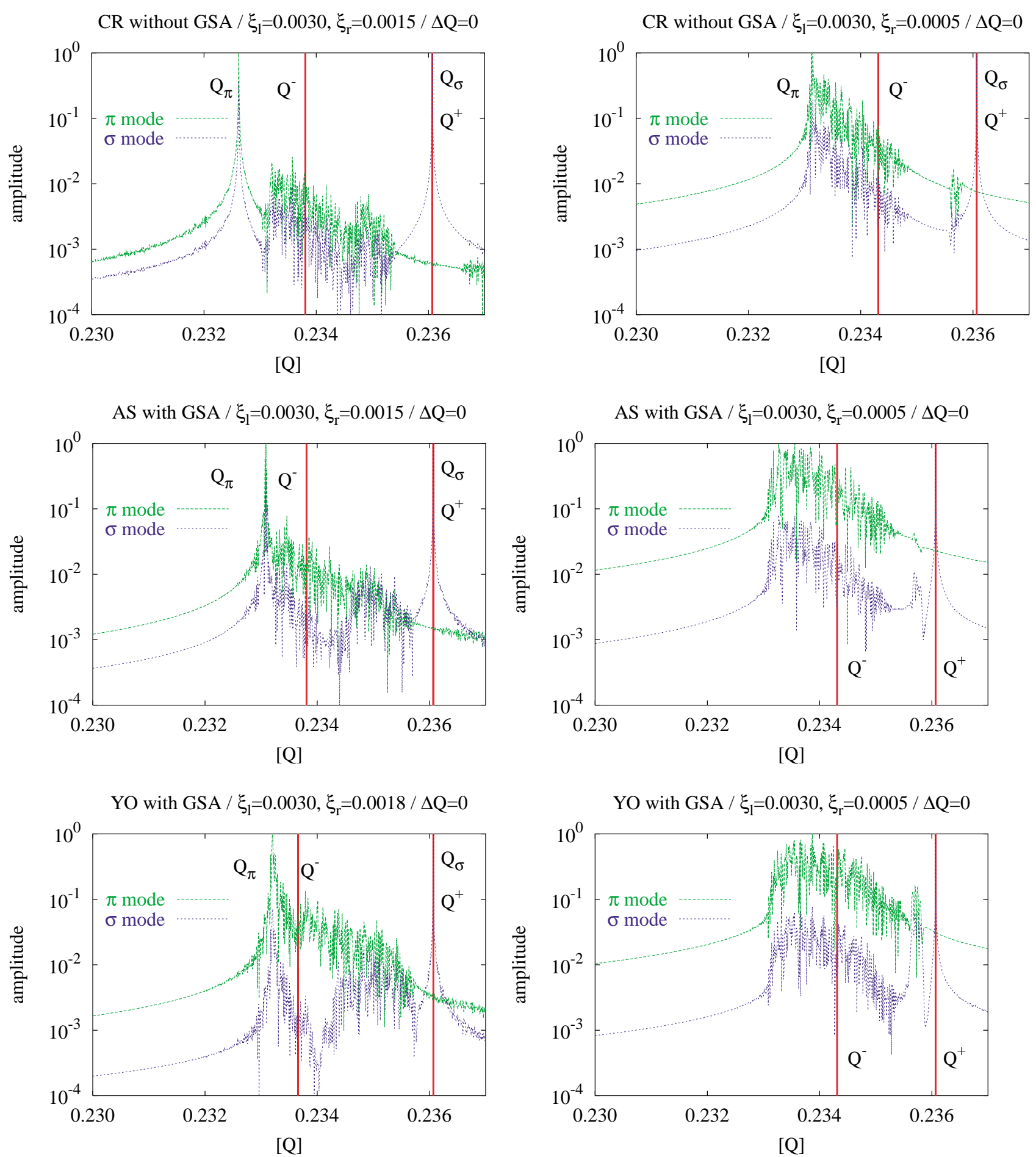

FIG. 8. (Color) FFT analysis of the $\pi$ and $\sigma$ modes for the three limits and for two ratios $\xi_{r} / \xi_{l}$ each (left) and 0.1667 (right). Top row: $\mathrm{CR}, \xi_{r} / \xi_{l}=0.5$ (left) and 0.1667 (right); center row: $\mathrm{AS}, \xi_{r} / \xi_{l}=0.5$ (left) and 0.1667 (right); bottom row: YO, $\xi_{r} / \xi_{l}=0.6$ (left) and 0.1667 (right).

$\xi_{r} / \xi_{l}=0.1667$ (right) then the $\pi$-mode peak has either disappeared completely (AS and YO) or is just a noisy maximum at the edge of the continuum (CR). In all three cases (CR, AS, and YO), the initial excitation is damped, accompanied by a slight emittance growth. In the case of $\mathrm{CR}$ we found that indeed a ratio of $\xi_{r} / \xi_{l}=0.1667$ was needed to move the $\pi$ mode at the edge of the continuum. At $\xi_{r} / \xi_{l}=0.5$ (top left) the Yokoya factor, now defined by $Y=2\left(Q_{\pi}-Q_{\sigma}\right) /\left(\xi+\xi^{*}\right)$, is 1.53 and even slightly higher than at $\xi_{r} / \xi_{l}=1$, where it is 1.51 .

In the AS limit, the $\pi$ mode merges with the continuum already at $\xi_{r} / \xi_{l}=0.5$ (middle left), but only little actual 
damping of the amplitudes or increase of the emittance (both not shown) was observed during the simulation. Actually both modes are clearly visible and we find $Y=1.33$. We note that at $\xi_{r} / \xi_{l}=0.6$ (not shown) the mode is still discrete. This result seems to be partly inconsistent with the predictions of $[5,23]$. However, it is consistent with the observation in Sec. V A that the Yokoya factor computed with WMPT and finite amplitude oscillations is slightly larger than the Yokoya factor computed using nonweighted macroparticle simulations or linearized Vlasov theory.

Finally, in the YO limit the $\pi$ mode merges with the continuum at $\xi_{r} / \xi_{l}=0.6$ (bottom left), but again the damping is only marginal and the Yokoya factor is 1.19 .

As expected, the intensity ratio at which the $\pi$ mode just emerges from the continuum depends on the model of the force. Furthermore, it seems that in order to attain efficient Landau damping the intensity ratio has to be well below this limit. The simulations presented in this section and in Sec. V A suggest for all three 1D limits that whenever the intensity ratio is decreased or the split of the bare tunes is increased, respectively, the Yokoya factor increases essentially until both eigenmodes disappear. In other words, the $\pi$-mode peak "flees" from the continuum before it is finally absorbed by continuum, and the eigenmodes are Landau damped.

\section{CONCLUSION AND OUTLOOK}

We studied the strong-strong beam-beam interaction by means of a method for simulating the evolution of the moments of the phase space distribution under collective Hamiltonian forces following the VE. This method, WMPT, has been implemented in a code for simulations, namely, BBDeMo1D.
Three different limits, the Chao-Ruth, the axially symmetric, and the Yokoya-Koiso-Zenkevich, have been studied numerically. The results in the CR case are in almost perfect agreement with independent simulations using the Perron-Frobenius operator technique [19,22] and (surprisingly) also the averaged/linearized Vlasov theory [20]. In the axially symmetric model our results agree very well with our more recent 2D WMPT simulations for round beams [22]. However, there is no complete quantitative agreement with the results of [23] using 2D conventional macroparticle tracking as well as with the linearized Vlasov theory [3-5]. We believe that the differences are due to the different types of phase space sampling. In the YO limit, which is basically accessible to WMPT only via the Gaussian source approximation, the simulations agree well with those performed in [6] and the discrepancy between the numerical results and the linearized Vlasov theory are seemingly due to the GSA [15].

The motion of the beam centroids under the collective force shows two dominant modes if the separation of the unperturbed tunes $\Delta Q$ is much smaller than the beam-beam tune shift parameter $\xi$. The modes are damped when $\Delta Q$ is significantly larger than $\xi$. The relative separation $\left(Q_{\sigma}-Q_{\pi}\right) / \xi$ depends not only on the model of the collective force but also on the separation of the unperturbed tunes $\Delta Q$. In the intermediate regime, the results depend on the type of the limit under consideration. Moreover, our simulations suggest that the modes are efficiently damped when the ratio $\xi / \xi^{*}$ differs strongly from 1 . For $\mathrm{CR}, \mathrm{AS}$, and YO the $\pi$ mode merges into the continuum at $\xi / \xi^{*} \approx 0.1667,0.5$, and 0.6 , respectively. The main results of Secs. V A and V B are summarized in Table II.

Only some aspects of the analysis have been presented. The way in which BBDeMo1D keeps track of and stores

TABLE II. Summary of results for CR, AS, and YO.

\begin{tabular}{|c|c|c|c|c|}
\hline Force & $\Delta Q$ & $\xi_{r} / \xi_{l}$ & $Q_{\sigma}-Q_{\pi}$ & Damping \\
\hline \multirow[t]{5}{*}{$\mathrm{CR}$} & 0 & 0.5 & $1.53\left(\xi_{l}+\xi_{r}\right) / 2$ & no \\
\hline & 0 & 0.1667 & $1.67\left(\xi_{l}+\xi_{r}\right) / 2^{\mathrm{a}}$ & yes \\
\hline & $5 \times 10^{-6}$ & 1 & $1.51 \xi$ & no \\
\hline & $1.5 \times 10^{-3}$ & 1 & $1.60 \xi$ & no \\
\hline & $6.0 \times 10^{-3}$ & 1 & $(2.61 \xi)^{\mathrm{a}}$ & yes \\
\hline \multirow[t]{5}{*}{ AS/GSA } & 0 & 0.5 & $1.33\left(\xi_{l}+\xi_{r}\right) / 2$ & $(y e s)^{b}$ \\
\hline & 0 & 0.1667 & $\ldots$ & yes \\
\hline & $5 \times 10^{-6}$ & 1 & $1.27 \xi$ & no \\
\hline & $1.5 \times 10^{-3}$ & 1 & $1.41 \xi$ & no \\
\hline & $6.0 \times 10^{-3}$ & 1 & $\cdots$ & yes \\
\hline \multirow{5}{*}{$\mathrm{YO} / \mathrm{GSA}$} & 0 & 0.6 & $1.19\left(\xi_{l}+\xi_{r}\right) / 2$ & $(y e s)^{b}$ \\
\hline & 0 & 0.1667 & $\ldots$ & yes \\
\hline & $5 \times 10^{-6}$ & 1 & $1.14 \xi$ & no \\
\hline & $0.5 \times 10^{-3}$ & 1 & $1.17 \xi$ & no \\
\hline & $6.0 \times 10^{-3}$ & 1 & $\ldots$ & yes \\
\hline
\end{tabular}

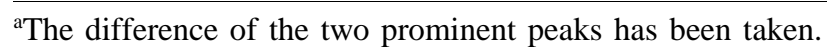

${ }^{\text {b}}$ The $\pi$ mode has reached the edge of the continuum but no or only weak reduction of the initial amplitudes is observed. 
different intermediate physical quantities (e.g., the macroparticle trajectories) allows a variety of postsimulation data analyses. Various postprocessing facilities, e.g., for the Fourier analysis of the trajectories and for frequency maps for the moments as well as the trajectories, have already been written and tested.

We extended the code to 2D motion. The basic structure of the code allows this to be done in a straightforward manner. Our first results for round beams are published in [22]. However, in order to efficiently handle the large number or macroparticles needed to grant a sufficiently dense sampling of the 4D phase space, it seems obligatory to parallelize the code, which will be our next step.

Furthermore, we plan to implement a more realistic representation of the lattice, long-range (parasitic) interactions, synchrotron motion, and, finally, more IPs and complex filling schemes.

\section{ACKNOWLEDGMENTS}

We gratefully acknowledge fruitful discussions with
Bob Warnock. One of the authors (M. V.) wishes to thank the members of the FNAL BD/BP group for the warm hospitality and fruitful cooperation during his stay at FNAL. The work of J.A.E. was supported by U.S. DOE Grant No. DE-FG03-99ER41104. The work of T.S. was supported by a U.S. DOE grant to Fermilab No. DE-AC02$76 \mathrm{HO} 3000$ and the U.S. LHC project. The work of M. V. was supported by U.S. DOE Grant No. DE-FG0399ER41104 and the U.S. LHC project.

\section{APPENDIX A: NUMERICAL REPRESENTATION OF THE YOKOYA-KOISO-ZENKEVICH FORCE}

In Sec. III C we noted that the kernel of the beam-beam kick in the Yokoya-Koiso-Zenkevich limit is singular and therefore its treatment in the WMPT approach is more critical than in the case of the Chao-Ruth limit and the axially symmetric limit. We start the discussion of the Yokoya-Koiso-Zenkevich limit by computing the beambeam kick, assuming we know $\psi_{\theta_{c}^{-}}^{*}$.

$$
K_{\mathrm{yo}}\left[\rho_{\theta_{c}^{-}}^{*}\right](q)=\lim _{\epsilon \searrow 0}\left(\int_{q+\epsilon}^{+\infty} \frac{\rho_{\theta_{c}^{-}}^{*}\left(q^{\prime}\right)}{q-q^{\prime}} d q^{\prime}+\int_{-\infty}^{q-\epsilon} \frac{\rho_{\theta_{c}^{-}}^{*}\left(q^{\prime}\right)}{q-q^{\prime}} d q^{\prime}\right)=\lim _{\epsilon \backslash 0} \int_{\mathbb{R}^{2}-\mathcal{A}_{q}(\epsilon)} \frac{\psi_{\theta_{c}^{-}}^{*}\left(\vec{z}^{\prime}\right)}{q-q^{\prime}} d^{2} z^{\prime}=I_{\eta}^{+}(q)+I_{\eta}^{-}(q),
$$

where

$$
I_{\eta}^{+}(q):=\int_{\mathbb{R}^{2}-\mathcal{A}_{q}(\eta)} \frac{\psi_{\theta_{c}^{-}}^{*}\left(\vec{z}^{\prime}\right)}{q-q^{\prime}} d^{2} z^{\prime}, \quad I_{\eta}^{-}(q):=\lim _{\epsilon \searrow 0} \int_{\mathcal{A}_{q}(\eta)-\mathcal{A}_{q}(\epsilon)} \frac{\psi_{\theta_{c}^{-}}^{*}\left(\vec{z}^{\prime}\right)}{q-q^{\prime}} d^{2} z^{\prime},
$$

and where $\mathcal{A}_{q}(x)=\mathbb{R} \times(q-x, q+x)$ is the (open) ribbon of width $2 x$ around $q$. In the last step we split the domain of integration into two parts. In the first one $\left[\mathbb{R}^{2}-\mathcal{A}_{q}(\eta) \rightarrow I_{\eta}^{+}\right]$we can perform an ordinary numeric quadrature, and in the second one $\left[\mathcal{A}_{q}(\eta)-\mathcal{A}_{q}(\epsilon) \rightarrow I_{\eta}^{-}\right]$with $\eta$ chosen suitably small, we can expand $\psi_{\theta_{c}^{-}}^{*}$ around $q$ w.r.t. $q^{\prime}$, $\left|q^{\prime}-q\right|<\eta$

$$
\psi_{\theta_{c}^{-}}^{*}\left(q^{\prime}, p^{\prime}\right)=\sum_{l=0}^{L} \frac{\psi^{(l)}\left(q, p^{\prime}\right)}{l !}\left(q^{\prime}-q\right)^{l}+O\left[\left(q^{\prime}-q\right)^{L+1}\right] .
$$

Since $\mathcal{A}_{g}(\eta)-\mathcal{A}_{g}(\epsilon)$ is symmetric around $q$, only the terms of $\psi_{\theta_{c}^{-}}^{*}\left(q^{\prime}\right) /\left(q-q^{\prime}\right)$ that are even in $q-q^{\prime}$ contribute to the integral, and we finally find

$$
K_{\mathrm{yo}}\left[\rho_{\theta_{c}^{-}}^{*}\right](q)=\int_{\mathbb{R}^{2}-\mathcal{A}_{q}(\eta)} \frac{\psi_{\theta_{c}^{-}}^{*}\left(\vec{z}^{\prime}\right)}{q-q^{\prime}} d^{2} z^{\prime}-2 \int_{\mathbb{R}} \sum_{l=0}^{(L-1) \operatorname{div} 2} \frac{\psi^{(2 l+1)}\left(q, p^{\prime}\right)}{(2 l+1) !(2 l+1)} \eta^{(2 l+1)} d p^{\prime}+O\left(\eta^{L^{\prime}}\right),
$$

where $L^{\prime}=L+2$ if $L$ is odd, and $L^{\prime}=L+1$ if $L$ is even. Thus, once $\psi_{\theta_{c}^{-}}^{*}$ is known and its derivatives w.r.t. $q^{\prime}$ can be computed, e.g., by divided differences, up to the presumably odd order $L$, the Cauchy principal value in $K_{\text {yo }}$ only adds to the overall numerical error of the integral to order $O\left(\eta^{L+2}\right)$, where $\eta$ is of the order of the (initial) mesh size. We note that in the case of known $\psi_{\theta_{c}^{-}}^{*}$ and correspondingly known $\rho_{\theta_{c}^{-}}^{*}$ we can formally simplify Eq. (A4),

$$
K_{\mathrm{yo}}\left[\rho_{\theta_{c}^{-}}^{*}\right](q)=\left(\int_{q+\eta}^{+\infty}+\int_{-\infty}^{q-\eta}\right) \frac{\rho_{\theta_{c}^{-}}^{*}\left(q^{\prime}\right)}{q-q^{\prime}} d q^{\prime}-2 \sum_{l=0}^{(L-1) \operatorname{div} 2} \frac{\rho^{(2 l+1)}(q)}{(2 l+1) !(2 l+1)} \eta^{(2 l+1)}+O\left(\eta^{L^{\prime}}\right),
$$

but we definitely need $\psi^{*}$ for WMPT. There we have

$$
\begin{aligned}
K_{\mathrm{yo}}\left[\rho_{\theta_{c}^{-}}^{*}\right](q) & =\lim _{\epsilon \backslash 0} \int_{\mathbb{R}^{2}-\mathcal{A}_{q}(\epsilon)} \frac{\psi_{0}^{*}\left(\vec{M}_{\theta_{c}^{-}}^{-1}\left(\vec{z}^{\prime}\right)\right)}{q-q^{\prime}} d^{2} z^{\prime}=\lim _{\epsilon \searrow 0} \int_{\mathbb{R}^{2}-\mathcal{B}_{q}(\epsilon)} \frac{\psi_{0}^{*}\left(\vec{z}^{\prime}\right)}{q-\mathfrak{X} \vec{M}_{\theta_{c}^{-}}(\vec{z})} d^{2} z^{\prime}, \\
& =\tilde{I}_{\eta}^{+}(q)+\tilde{I}_{\eta}^{-}(q),
\end{aligned}
$$


where

$$
\begin{aligned}
& \tilde{I}_{\eta}^{+}(q):=\int_{\mathbb{R}^{2}-\mathcal{B}_{q}(\eta)} \frac{\psi_{0}^{*}\left(\vec{z}^{\prime}\right)}{q-\mathfrak{X} \vec{M}_{\theta_{c}^{-}}\left(\vec{z}^{\prime}\right)} d^{2} z^{\prime}, \\
& \tilde{I}_{\eta}^{-}(q):=\lim _{\epsilon \searrow 0} \int_{\mathcal{B}_{q}(\eta)-\mathcal{B}_{q}(\epsilon)} \frac{\psi_{0}^{*}\left(\vec{z}^{\prime}\right)}{q-\mathfrak{X} \vec{M}_{\theta_{c}^{-}}\left(\vec{z}^{\prime}\right)} d^{2} z^{\prime},
\end{aligned}
$$

and where $\mathcal{B}_{q}(x):=\vec{M}_{\theta_{c}^{-}}^{-1}\left(\mathcal{A}_{q}(x)\right)$ is the subset of $\mathbb{R}^{2}$ so that for all $\vec{z} \in \mathcal{B}_{q}(x): \vec{M}_{\theta_{c}^{-}}(\vec{z}) \in \mathcal{A}_{q}$ and $\mathfrak{X} \vec{z}$ is the projection of $\vec{z}$ onto configuration space. The integral $\tilde{I}_{\eta}^{+}(q)=I_{\eta}^{+}(q)$ does not produce any algorithmic complication. It is given as the sum over all the weighted macroparticles that at $\theta_{c}^{-}$fall outside of $\mathcal{A}_{q}(\eta)$. But $\tilde{I}_{\eta}^{-}(q)$ is an integral that not only can have a fairly complicated domain due to the nonlinearity of $\vec{M}_{\theta}$, but the projection $\tilde{\eta}$ of the set $\mathcal{B}_{q}(\eta)$ on the $q$ axis even for arbitrarily small $\eta$ cannot be guaranteed to be small. Therefore the truncated Taylor expansion Eq. (A3) that leads to a good approximation of $I_{\eta}^{-}(q)$ for small $\eta$ cannot be applied to $\tilde{I}_{\eta}^{-}(q)$.

Note that mathematically Eq. (A6) is still well defined since, because of the volume preservation of $\vec{M}_{\theta}$, in the limit $\epsilon \rightarrow 0$ with the measure of $\mathcal{A}_{q}(\epsilon)$ also the measure of $\mathcal{B}_{q}(\epsilon)$ goes to zero. Just its numerical representation has an error which cannot so easily be bounded.

Therefore in WMPT, the integral $\tilde{I}_{\eta}^{-}(q)$ is not well represented. In the current version of BBDeMolD it is just approximated by $\tilde{I}_{\eta}^{-}(q)=0$ with a reasonably small $\eta$. The reason that $\eta$ cannot be chosen arbitrarily small is that in order to get a good approximation of the Cauchy principle value one needs sufficiently large and basically equal relative densities of many macroparticles on both sides but close to the boundary of $\mathcal{A}_{q}(\eta)$. Otherwise, single particles being close to the boundary and not balanced by a particle "on the other side" will produce a strong and basically stochastic kick. It turned out during the simulation that the approximation $K_{\mathrm{yo}}\left[\rho_{\theta_{c}^{-}}^{*}\right](q) \approx \tilde{I}_{\eta}^{+}(q)$ destabilizes the beam in the used parameter range (see Sec. IV). It has to be noted that the PF method, since it does have knowledge of a numerical approximation of $\psi_{\theta_{c}^{-}}^{*}$, should in principle be more suitable to handle the Yokoya-KoisoZenkevich force.

\section{APPENDIX B: THE GSA FOR THE YOKOYA- KOISO-ZENKEVICH FORCE}

The GSA for the Yokoya-Koiso-Zenkevich force leads to

$$
K_{\mathrm{yo}}[\rho](x)=\frac{\zeta}{\sqrt{2 \pi} \sigma} f \frac{e^{-\left[\left(x^{\prime}-\mu\right)^{2} / 2 \sigma^{2}\right]}}{x-x^{\prime}} d x^{\prime} .
$$

We now compute the Cauchy principle value using methods from complex variables.

We want to compute

$$
f(x):=\frac{1}{\sqrt{\pi}} f \frac{e^{-s^{2}}}{x-s} d s .
$$

We consider the function

$$
G(z):=\frac{1}{2 \pi i} \int_{\mathbb{R}} \frac{e^{-s^{2}}}{s-z} d s .
$$

Clearly, $G$ is holomorphic for $\Im z \neq 0$. Now let $z=$ : $x+i y$. Then using Plemelj's formula [26] we find

$$
G\left(x+i 0^{ \pm}\right)= \pm \frac{1}{2} e^{-x^{2}}+\frac{1}{2 \pi i} f \frac{e^{-s^{2}}}{s-z} d s .
$$

We now define

$$
\begin{aligned}
F(z) & :=G(z), \quad \Im z<0, \\
& :=-\frac{1}{2} e^{-x^{2}}-\frac{1}{2 \sqrt{\pi} i} f(x), \quad \Im z=0, \\
& :=-e^{-z^{2}}+G(z), \quad \Im z>0 .
\end{aligned}
$$

Note that $F$ is holomorphic for $\Im z \neq 0$ and continuous for $\Im z=0$. It follows (see, e.g., theorem 3.2.7 of [26]) that $F$ is entire. Moreover, note that

$$
F(z)=\frac{1}{2 \pi i} \int_{C} \frac{e^{-s^{2}}}{s-z} d s,
$$

where $C$ is a Landau contour described in Fig. 9.

Differentiating $G$ for $\Im z<0$, integrating by parts, and using $s /(s-z)=z /(s-z)+1$, we find

$$
\begin{aligned}
G^{\prime}(z) & =\frac{1}{2 \pi i} \int_{\mathbb{R}} \frac{e^{-s^{2}}}{(s-z)^{2}} d s \\
& =\frac{1}{2 \pi i}\left(\left.\frac{-e^{-s^{2}}}{s-z}\right|_{-\infty} ^{+\infty}-\int_{\mathbb{R}} \frac{2 s e^{-s^{2}}}{s-z} d s\right) \\
& =\frac{-1}{\pi i} \int_{\mathbb{R}}\left(\frac{e^{-s^{2}}}{s-z}+e^{-s^{2}}\right) d s \\
& =-2 z G(z)-\frac{1}{\sqrt{\pi} i} .
\end{aligned}
$$
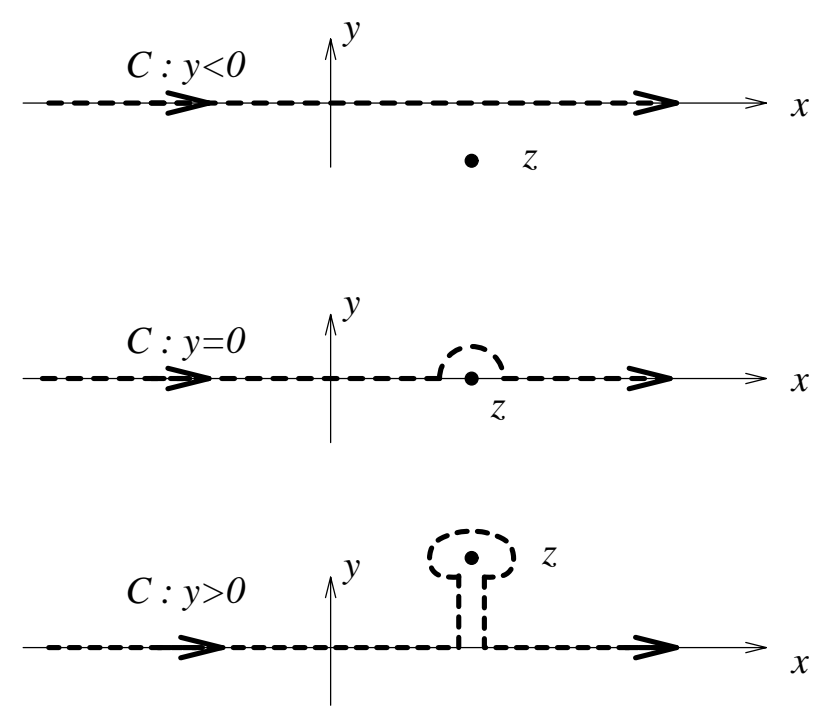

FIG. 9. The Landau contour. 
It follows that

$$
F^{\prime}+2 z F=\frac{i}{\sqrt{\pi}}
$$

for all $z \in \mathbb{C}$ since, if two entire functions are equal on an open subset of $\mathbb{C}$, they are equal on $\mathbb{C}$. Now because of Eqs. (B6) and (B2), and since $e^{-s^{2}}$ is even whereas $1 / s$ is odd, we find that $f$ is a solution of the initial value problem

$$
f^{\prime}+2 x f=2, \quad f(0)=0 .
$$

\begin{tabular}{|c|c|}
\hline Symbol & Comment \\
\hline$\theta$ & Generalized machine azimuth \\
\hline$\theta_{c}$ & Azimuth of collision point \\
\hline$f\left(\theta_{c}^{-}\right), f\left(\theta_{c}^{+}\right)$ & $\lim _{\theta / \theta_{c}} f(\theta), \lim _{\theta \searrow \theta_{c}} f(\theta)$ \\
\hline$q, x, y, r$ & $\begin{array}{l}\text { Spatial coordinate (general, horizontal, } \\
\text { vertical, radial) }\end{array}$ \\
\hline$p$ & Conjugate momentum $[\vec{z} \equiv(q, p)]$ \\
\hline$\sigma_{0}$ & Initial rms beam width \\
\hline$\psi_{\theta}(q, p)$ & Phase space density (normalized to 1 ) at $\theta$ \\
\hline$\rho_{\theta}(q)$ & Spatial density (normalized to 1 ) at $\theta$ \\
\hline$\tilde{\rho}(q ;\{$ parameters $\})$ & Test density $(\rightarrow$ GSA $)$ \\
\hline$\rho(q ; \mu, \sigma)$ & $\begin{array}{l}\text { Gaussian with } \\
\qquad \mu:=\langle q\rangle, \sigma^{2}:=\left\langle(q-\mu)^{2}\right\rangle\end{array}$ \\
\hline$\vec{R}$ & Linear lattice map \\
\hline$\underline{R}$ & Jacobian of linear lattice map \\
\hline$Q_{0}, \beta_{0}, \alpha_{0}, \gamma_{0}$ & $\begin{array}{l}\text { Tune and Courant-Snyder functions } \\
\text { due to linear lattice }\end{array}$ \\
\hline$\vec{K}$ & Beam-beam kick map \\
\hline$K[\rho](q)$ & Kick function, i.e., $p \rightarrow p+K[\rho](q)$ \\
\hline$\tilde{G}\left(q, q^{\prime}\right)$ & Green's function of Poisson equation \\
\hline$G\left(q, q^{\prime}\right)$ & {$\left[\mathrm{CR} / \mathrm{YO}: \equiv \partial_{q} G\left(q, q^{\prime}\right)\right]$ beam-beam kernel } \\
\hline$\tilde{K}(q ;\{$ parameters $\})$ & Kick function using test density ( $\rightarrow$ GSA) \\
\hline$\kappa\left[\frac{K}{\rho]}(q)\right.$ & $\begin{array}{l}\text { Jacobian of beam-beam kick map } \\
\equiv(K)_{21} \text { linearized kick function, } \\
\quad \text { i.e., } K[\rho](q)=\kappa[\rho] q+O\left(q^{2}\right)\end{array}$ \\
\hline$\xi$ & Linear beam-beam tune shift parameter \\
\hline$\zeta$ & $\begin{array}{l}\equiv \zeta(\xi) \text { proportionality factor } \\
\text { in beam-beam kick }\end{array}$ \\
\hline$\vec{T}$ & One turn map \\
\hline$T$ & Jacobian of one turn map \\
\hline$\vec{M}_{\theta}$ & Map from 0 to $\theta$ \\
\hline $\mathfrak{I}$ & Perron-Frobenius operator \\
\hline $\mathfrak{X}$ & Projector on spatial coordinate(s) \\
\hline$\vec{z}_{i j}$ & $\equiv\left(q_{i}, p_{j}\right)$ mesh point \\
\hline$\vec{\eta}_{i j}(\theta)$ & $\equiv \vec{M}_{\theta}\left(\vec{z}_{i j}\right)$ trajectory starting at mesh point \\
\hline $\mathfrak{W}_{i j}$ & Total weight of a trajectory \\
\hline$\chi\left(\mu, q, q^{\prime}\right)$ & Indicator function of $[\mu-q, \mu+q]$ \\
\hline$\chi_{\mu, \nu}(\vec{z})$ & Indicator function of bin $(\mu, \nu)$ \\
\hline$n$ & $\begin{array}{l}\text { Number of particles per phase space } \\
\text { dimension }\end{array}$ \\
\hline$N$ & $\begin{array}{l}\text { Number of particles in initial mesh } \\
\left(\equiv n^{2} \text { for one dof }\right)\end{array}$ \\
\hline $\mathbb{R}, \mathbb{R}^{2}$ & Configuration space, phase space \\
\hline $\mathcal{A}$ & Some set (e.g., $\mathcal{A} \subset \mathbb{R}^{2}$, etc.) \\
\hline$f$ & Cauchy principal value \\
\hline $\begin{array}{c}a:=b, a=: b \\
a \equiv b\end{array}$ & $\begin{array}{l}a \text { defined by } b, b \text { defined by } a \\
a \text { and } b \text { identical by definition }\end{array}$ \\
\hline
\end{tabular}

TABLE III. Symbols used in this paper.
Note that if one differentiates $f$ defined by Eq. (B2) and then "bravely" interchanges the differentiation with the limit of the Cauchy principle value, one obtains Eq. (B11) following the procedure in Eq. (B9).

One easily verifies that

$$
f(x)=2 e^{-x^{2}} \int_{0^{x}} e^{t^{2}} d t=\sqrt{\pi} \Im W(x),
$$

where $\mathrm{W}$ is the complex "error" function $[17,18]$, solves Eq. (B11).

Finally, replacing $x$ by $\left(x-\mu^{*}\right) /\left(\sigma^{*} \sqrt{2}\right)$ yields the expression in Eq. (39).

\section{APPENDIX C: CONVENTIONS AND USED SYMBOLS}

Let $X$ be a quantity of one beam, then $X^{*}$ is the same quantity for the other beam. A more old-fashioned notation would be using $X^{(1)}$ and $X^{(2)}$. We want to stress the point that the beam-beam interaction is formally symmetric and thus from any equation including lattice and beam-beam effects for one beam we immediately obtain the corresponding equation for the other beam by toggling the asterisk on all relevant parameters and dynamical variables. Table III summarizes the definitions of most of the symbols used in this paper.

[1] S. K. Kauffmann et al., in Advanced Beam Dynamics Workshop on Effects of Errors in Accelerators, edited by A. Chao, AIP Conf. Proc. No. 255 (AIP, New York, 1992).

[2] A. W. Chao and R.D. Ruth, Part. Accel. 16, 201-216 (1985).

[3] K. Yokoya and H. Koiso, Part. Accel. 27, 181-186 (1990).

[4] K. Yokoya and P. Zenkevich, Part. Accel. 40, 229-241 (1993).

[5] Y. Alexahin, Part. Accel. 59, 43-74 (1998).

[6] M.-P. Zorzano and F. Zimmermann, CERN LHC Project Report No. 314, CERN/SL, 1999.

[7] E. B. Anderson, Ph.D thesis, Cornell University, 1998.

[8] S. Krishnagopal, Phys. Rev. ST Accel. Beams 3, 024401 (2000).

[9] R. L. Warnock and J. A. Ellison, in Proceedings of the 2nd ICFA Workshop on High Brightness Beams, UCLA, 1999, edited by J. Rosenzweig and L. Serafini (World Scientific, Singapore, 2000).

[10] J. A. Ellison and R. L. Warnock, in Proceedings of the 2nd ICFA Workshop on Quantum Aspects of Beam Physics, Capri, 2000 (World Scientific, Singapore, 2000).

[11] S. Wollman and E. Ozizmir, SIAM J. Numer. Anal. 33, 1377-1409 (1996).

[12] S. Wollman, J. Comput. Appl. Math. 115, 593-600 (1999).

[13] S. Wollman, SIAM J. Numer. Anal. 37, 1369-1398 (2000).

[14] M. A. Furman, in Proceedings of the International Computational Accelerator Physics Conference, Monterey, 1998, http://www.slac.stanford.edu/xorg/icap98/abst.htm\# Focused8.

[15] K. Yokoya, Phys. Rev. ST Accel. Beams 3, 124401 ( 2000). 
[16] W.H. Press, S. A. Teukolsky, W. T. Vetterling, and B.P. Flannery, Numerical Recipes in FORTRAN (Cambridge University, Cambridge, 1992), 2nd ed.

[17] I. Gradstein and I. Ryshik, Tables of Series, Products and Integrals (Verlag Harri Deutsch, Frankfurt am Main, 1981).

[18] CERN, CERNLIB, short write ups, http://wwwinfo.cern.ch/ asdoc/cernlib.html.

[19] J. A. Ellison, M. Vogt, and T. Sen, in Proceedings of the Particle Accelerator Conference, Chicago 2001 (to be published).

[20] J. A. Ellison and M. Vogt, in Proceedings of the BeamBeam Workshop at Fermilab, 2001, http://www-ap.fnal. gov/ meiqin/beambeam01/beambeam01.html

[21] A. Hofmann, in Proceedings of the Large Hadron Collider
(LHC99) Beam-Beam Workshop, 1999 (CERN, Geneva, 1999), pp. 56-58.

[22] M. Vogt, J. A. Ellison, T. Sen, and R. Warnock, in Proceedings of the Beam-Beam Workshop at Fermilab, 2001 (Ref. [20]).

[23] W. Herr, M.-P. Zorzano, and F. Jones, Phys. Rev. ST Accel. Beams 4, 054402 (2001).

[24] F. W. Jones, in Workshop on Space Charge Physics in High Intensity Hadron Rings, edited by A. U. Luccio and W. T. Weng, AIP Conf. Proc. No. 448 (AIP, Piscataway, NJ, 1998).

[25] L. Greengard, Ph.D. thesis, MIT, 1988.

[26] M. J. Ablowitz and A. S. Fokas, Complex Variables (Cambridge University, Cambridge, 1997). 